\title{
UNIFORM ANALYTICITY OF ORTHOGONAL PROJECTIONS
}

\author{
R. R. COIFMAN AND M. A. M. MURRAY
}

\begin{abstract}
Let $X$ denote the circle $T$ or the interval $[-1,1]$, and let $d \mu$ denote a nonnegative, absolutely continuous measure on $X$. Under what conditions does the Gram-Schmidt procedure in the weighted space $L^{2}\left(X, \omega^{2} d \mu\right)$ depend analytically on the logarithm of the weight function $\omega$ ? In this paper, we show that, in numerous examples of interest, $\log \omega \in B M O$ is a sufficient (often necessary!) condition for analyticity of the Gram-Schmidt procedure. These results are then applied to establish the local analyticity of certain infinitedimensional Toda flows.
\end{abstract}

\section{INTRODUCTION}

Let $X$ denote the circle $\mathbf{T}$ or the interval $[-1,1]$, let $d \mu$ be a nonnegative measure on $X$ which is absolutely continuous with respect to Lebesgue measure, and let $L(0)$ denote the complex Hilbert space $L^{2}(X, d \mu)$. Let $\omega$ be a nonnegative $d \mu$-measurable function on $X$ such that $\omega^{2}+\omega^{-2} \in L^{1}(X, d \mu)$ and let $\beta=\log \omega$; clearly also $\beta \in L^{1}(X, d \mu)$. Let $L(\beta)$ denote the complex weighted Hilbert space $L^{2}\left(X, \omega^{2} d \mu\right)$ and, for each nonnegative integer $n$, let $H_{n}(\beta)$ denote the closure of the polynomials (in the case $X=\mathrm{T}$, trigonometric polynomials) of degree at most $n$ in $L(\beta)$. Let $S_{n}(\beta)$ denote the orthogonal projection of $L(\beta)$ onto $H_{n}(\beta)$. We wish to study the dependence of the family of operators $\left\langle S_{n}(\beta): n \in \mathbf{N}\right\rangle$ upon the functional parameter $\beta$. Each $S_{n}(\beta)$ is a bounded operator on $L(\beta)$, which varies with $\beta$, so to facilitate our study, we "lift" each operator $S_{n}(\beta)$ to $L(0)$ by means of the operator $M_{\omega}$ of pointwise multiplication by $\omega$, which is an isometry from $L(\beta)$ to $L(0)$. If we define, for each nonnegative integer $n$, the operator $Q_{n}(\beta)=M_{\omega} S_{n}(\beta) M_{\omega}^{-1}$, then we see that the $L(0)$-boundedness of $Q_{n}(\beta)$ is equivalent to the $L(\beta)$ boundedness of $S_{n}(\beta)$, and the operator norms are equal. In fact, $Q_{n}(\beta)$ is easily seen to be the self-adjoint projection of $L(0)$ onto $M_{\omega} H_{n}(\beta) \subseteq L(0)$.

We would like to determine conditions on $\beta$ under which the family of operators $\left\langle Q_{n}(\beta)\right\rangle$ depends analytically (in a sense to be made precise) upon the functional parameter $\beta$. In the specific examples which we consider, it is

Received by the editors December 1, 1987.

1980 Mathematics Subject Classification (1985 Revision). Primary 42A05, 42C05, 33A65, 42C10, 46G20, 47A55, 47B38.

Both authors partially supported by grants from the National Science Foundation. 
difficult if not impossible to write down the operators $S_{n}(\beta)$ and $Q_{n}(\beta)$ explicitly. On the other hand, the "base projection" $S_{n}(0)$ is an integral operator whose kernel is comparatively easy to write down. Rather than study the operators $\left\langle Q_{n}(\beta)\right\rangle$ directly, it is much more convenient to work with the family of operators $\left\langle P_{n}(\beta)\right\rangle$ defined by

$$
P_{n}(\beta)=M_{\omega} S_{n}(0) M_{\omega}^{-1} .
$$

For each nonnegative integer $n, P_{n}(\beta)$ is an oblique (i.e., non-self-adjoint) projection from $L(0)$ onto $M_{\omega} H_{n}(\beta) \subseteq L(0)$, and its adjoint, $P_{n}(\beta)^{*}$, is simply $P_{n}(-\beta)$.

A remarkable formula due to Kerzman and Stein ([8]) shows that, in fact, $\left\langle Q_{n}(\beta)\right\rangle$ depends analytically on $\beta$ whenever $\left\langle P_{n}(\beta)\right\rangle$ depends analytically on $\beta$. Moreover, the analytic dependence of $\left\langle P_{n}(\beta)\right\rangle$ upon $\beta$ is essentially equivalent to a uniform weighted norm inequality of the form

$$
\int_{X}\left|S_{n}(0) f(x)\right|^{2} \omega^{2}(x) d \mu(x) \leq C \int_{X}|f(x)|^{2} \omega^{2}(x) d \mu(x)
$$

where $C$ is a constant independent of $n$ and $f$.

In addition, we would like to determine the "space of uniform holomorphy" for the family $\left\langle Q_{n}(\beta)\right\rangle$, i.e. the largest Banach function space on which the family $\left\langle Q_{n}\right\rangle$ is analytic at the origin. In practice, this amounts to determining necessary and sufficient conditions on the function $\beta$ such that

$$
\int_{X}\left|\left[M_{\beta}, S_{n}(0)\right] f(x)\right|^{2} d \mu(x) \leq C \int_{X}|f(x)|^{2} d \mu(x)
$$

where $M_{\beta}$ is the operator of pointwise multiplication by $\beta$, and $C$ is a constant independent of $n$ and $f$.

Our work in this paper has been inspired in part by the related work of Coifman and Rochberg in [2], and by questions arising from the study of Toda flows in infinite dimensions (see, for example, [4]).

In this paper, we consider a number of examples. In the case $X=\mathbf{T}, d \mu=$ $d \theta$, the uniform weighted norm inequality (1.2), and the uniform commutator estimate (1.3), are equivalent to the same inequalities with $S_{n}(0)$ replaced by the conjugate function. In this simplest example, the space of uniform holomorphy for $\left\langle Q_{n}(\beta)\right\rangle$ is easily seen to be the space of functions of bounded mean oscillation on $\mathbf{T}$. In the case $X=[-1,1], d \mu=$ Lebesgue measure weighted by a Jacobi weight, the uniform weighted norm inequality (1.2) follows from a weighted norm inequality for the Hilbert transform. In this case, we prove that $\left\langle Q_{n}(\beta)\right\rangle$ depends analytically on $\beta$ when $\beta$ is in a neighborhood of 0 in the space $\mathbf{B M O}([-1,1])$. As an application of this result, we show that the Toda flow corresponding to the measure $\omega(x)^{2 t} d \mu(x)$ on $[-1,1]$ is analytic in $t$ in a neighborhood of the origin provided that $\beta \in \mathbf{B M O}([-1,1])$.

We conjecture that $\mathbf{B M O}([-1,1])$ is the space of uniform holomorphy for $\left\langle Q_{n}\right\rangle$ in the case where $d \mu=$ Lebesgue measure weighted by a Jacobi weight. 
We consider the example $X=[0, \pi], d \mu=d \theta, S_{n}=n$th partial sum operator for cosine series, and show that, in this example, $\operatorname{BMO}([0, \pi])$ is the space of uniform holomorphy for $\left\langle Q_{n}\right\rangle$. From this result it is immediate that the conjecture is true for $d \mu(x)=\left(1-x^{2}\right)^{-1 / 2} d x$. Classical equiconvergence results for Jacobi series and cosine series (see [10]) suggest that the conjecture is probably true in general.

\section{UNIFORM ANALYTICITY OF PROJECTIONS: GENERAL SETTING}

Let $(X, d \mu)$ be a $\sigma$-finite measure space; set $L(0)=L^{2}(X, d \mu)$. Suppose that $\omega$ is a nonnegative real-valued function such that $\omega^{2}+\omega^{-2} \in L_{\text {loc }}^{1}(X, d \mu)$. We write $\beta=\log \omega$ and observe that $\beta \in L_{\text {loc }}^{1}(X, d \mu)$. Let $\mathbf{N}$ denote the set of nonnegative integers, and suppose that for each $n \in \mathbf{N}, H_{n}(0)$ is a closed subspace of $L(0)$. We assume that

$L(0, \beta)=L^{2}\left(X,\left(\omega^{2}+\omega^{-2}\right) d \mu\right)$ is dense in $L(0)$ and

$H_{n}(0, \beta)=L(0, \beta) \cap H_{n}(0)$ is dense in $H_{n}(0)$, for each $n \in \mathbf{N}$.

We define the spaces:

$L(\beta)=L^{2}\left(X, \omega^{2} d \mu\right)$,

$\mathscr{L}(\beta)=$ bounded linear operators on $L(\beta)$,

$H_{n}(\beta)=$ closure of $H_{n}(0, \beta)$ in $L(\beta)$, for each $n \in \mathbf{N}$.

We assume that the foregoing are complex Hilbert spaces.

For each $n \in \mathbf{N}$, let $S_{n}(\beta) \in \mathscr{L}(\beta)$ be the selfadjoint projection of $L(\beta)$ onto $H_{n}(\beta)$. We wish to study the dependence of the operators $\left\langle S_{n}(\beta): n \in\right.$ N) upon the functional parameter $\beta$. To facilitate our study, we "lift" each operator $S_{n}(\beta)$ back to $\mathscr{L}(0)$ by means of the operator $M_{\omega}$ of pointwise multiplication by $\omega$, to wit: for each $n \in \mathbf{N}$, define $Q_{n}(\beta)=M_{\omega} S_{n}(\beta) M_{\omega}^{-1}$. Then $Q_{n}(\beta)$ is the self-adjoint projection of $L(0)$ onto $M_{\omega} H_{n}(\beta) \subseteq L(0)$, and $\left\|Q_{n}(\beta)\right\|_{\mathscr{L}(0)}=\left\|S_{n}(\beta)\right\|_{\mathscr{L}(\beta)}$.

We would like to formulate a clear conception of the "analytic dependence" of the family of operators $\left\langle Q_{n}(\beta): n \in \mathbf{N}\right\rangle$ upon the functional parameter $\beta$. To this end, we make the following definitions.

Definitions. Let $B$ be a real Banach space, $\mathscr{L}(H)$ the space of bounded linear operators on a complex Hilbert space $H$. For $n \in \mathbf{N}$, let $T_{n}: B \rightarrow \mathscr{L}(H)$ be an operator-valued function on $B$.

(a) $\left\langle T_{n}: n \in \mathbf{N}\right\rangle$ is said to be uniformly (real-) analytic in a neighborhood of 0 in $B$ if and only if there is a constant $C>0$ such that, whenever $b \in B$ with $\|b\|_{B} \leq C$ and whenever $f \in H$, we have

$$
T_{n}(b) f=\sum_{k=0}^{\infty} \Lambda_{n, k}(b, \ldots, b, f), \quad \text { for all } n \in \mathbf{N},
$$

where $\Lambda_{n, k}$ is a bounded, $(k+1)$-multilinear operator from $B^{k} \times H \rightarrow H$ which satisfies an estimate of form

$$
\left\|\Lambda_{n, k}(b, \ldots, b, f)\right\|_{H} \leq C_{0}^{k+1}\|b\|_{B}^{k}\|f\|_{H}
$$

where $C_{0}$ is independent of $b, f, n$, and $k$. 
(b) Let $\mathbf{B}$ denote the complexification of $B .\left\langle T_{n}: n \in \mathbf{N}\right\rangle$ is said to be uniformly holomorphic in a neighborhood of 0 in $\mathbf{B}$ if and only if there is a neighborhood of 0 in $\mathbf{B}$ to which each $T_{n}$ can be extended, and there is a constant $C>0$ such that, whenever $b \in \mathbf{B}$ with $\|b\|_{\mathbf{B}} \leq C$ and whenever $f \in H$, we have (2.1) and (2.2) with ' $\mathbf{B}$ ' in place of ' $B$ '.

(c) $\mathbf{B}$ is called the space of uniform holomorphy at 0 for the family $\left\langle T_{n}: n \in\right.$ $\mathbf{N}\rangle$ if and only if $\left\langle T_{n}\right\rangle$ is uniformly holomorphic in a neighborhood of 0 in $\mathbf{B}$ and a necessary and sufficient condition for

$$
\sup _{n}\left\|\Lambda_{n, 1}(b, \cdot)\right\|_{\mathscr{L}(H)}
$$

to be finite is that $b \in \mathbf{B}$.

We pause to observe that the operator $\Lambda_{n, k}(b, \ldots, b, \cdot)$ occurring in (2.1) is just the $k$ th Gâteaux (or Frechét) differential of $T_{n}$ at 0 in the direction $b$ (see, for example, [1, Chapter 2]). We give one equivalent formulation of the notion of uniform holomorphy in terms of Gâteaux differentiability, which will be useful in practice.

Proposition 2.1. Let B be a complex Banach space, $\mathscr{L}(H)$ the space of bounded linear operators on a complex Hilbert space $H$. For $n \in \mathbf{N}$, let $T_{n}: \mathbf{B} \rightarrow \mathscr{L}(H)$ be an operator-valued function on $\mathbf{B}$. Then $\left\langle T_{n}\right\rangle$ is uniformly holomorphic in a neighborhood of 0 in $\mathbf{B}$ if and only if there exists a neighborhood $U$ of 0 in $\mathbf{B}$ on which each $T_{n}$ is Gâteaux differentiable and there exists a constant $C$ such that for all $n \in \mathbf{N}$ and for all $\beta \in U,\left\|T_{n}(\beta)\right\|_{\mathscr{L}(H)} \leq C$.

Proof. The proof is an easy modification of the proof of Theorem 2.3.3 of [1] and will be omitted.

We can now formulate our general problem precisely, as follows: we wish to identify the space of uniform holomorphy at 0 for the family $\left\langle Q_{n}: n \in \mathbf{N}\right\rangle$. In practice it is often difficult to characterize the projections $S_{n}(\beta)$ and $Q_{n}(\beta)$; it is much more convenient to work with the operators $\left\langle P_{n}(\beta): n \in \mathbf{N}\right\rangle$ defined by

$$
P_{n}(\beta)=M_{\omega} S_{n}(0) M_{\omega}^{-1} .
$$

For $n \in \mathbf{N}, P_{n}(\beta)$ is an oblique projection from $L(0)$ onto $M_{\omega} H_{n}(\beta)$ with adjoint $P_{n}(\beta)^{*}=P_{n}(-\beta)$. The formula of Kerzman and Stein (see $[8, \S 3.4]$ ) makes it possible to deduce the uniform holomorphy of $\left\langle Q_{n}\right\rangle$ from that of $\left\langle P_{n}\right\rangle$, and greatly simplifies the computation of the Gâteaux differentials of the operators $\left\langle Q_{n}\right\rangle$ :

Proposition 2.2 (Kerzman-Stein Formula). Let $H$ be a complex Hilbert space, $K$ a closed subspace. Let $Q$ be the self-adjoint projection of $H$ onto $K$, and let $P$ be a bounded oblique projection from $H$ onto $K$. Then:

(a) $I+\left(P-P^{*}\right)$ is invertible.

(b) $Q=P\left[I+\left(P-P^{*}\right)\right]^{-1}$; 
(c) whenever $c_{0}$ and $M$ are positive constants with $\left\|P-P^{*}\right\| \leq c_{0}$ and $M>\frac{1}{2}\left(c_{0}^{2}-1\right)$, the series

$$
P\left\{\frac{1}{M+1} \sum_{k=0}^{\infty}\left[\frac{M I-\left(P-P^{*}\right)}{M+1}\right]^{k}\right\}
$$

converges in the operator norm topology to $Q$.

Proof. The operator $P-P^{*}$ is skew-adjoint, so its spectrum is purely imaginary. In particular, -1 is not in the spectrum of $P-P^{*}$, from which (a) follows.

Clearly $Q P=P$ and $P Q=Q$. Now let $h \in H$ and let $(\cdot \cdot \cdot)$ denote the inner product on $H$. We have

$$
\begin{aligned}
\left(Q P^{*} h \mid h\right) & =\left(P^{*} h \mid Q h\right) \quad \text { since } Q^{*}=Q \\
& =(h \mid P Q h) \\
& =(h \mid Q h) \quad \text { since } P Q=Q \\
& =(Q h \mid h) \quad \text { since } Q^{*}=Q .
\end{aligned}
$$

Hence $Q P^{*}=Q$; consequently

$$
P=Q+(P-Q)=Q+\left(Q P-Q P^{*}\right)=Q\left[I+\left(P-P^{*}\right)\right]
$$

whereupon we obtain (b).

It is tempting to expand $\left[I+\left(P-P^{*}\right)\right]^{-1}$ in a Neumann series, but we do not know that $\left\|P-P^{*}\right\|<1$. Instead we proceed as follows. For any constant $M>0$, we have

$$
\begin{aligned}
I+\left(P-P^{*}\right) & =I+M I-\left[M I-\left(P-P^{*}\right)\right] \\
& =(1+M)\left[I-\frac{M I-\left(P-P^{*}\right)}{1+M}\right] .
\end{aligned}
$$

Recall that, if $S^{*}=S, T^{*}=-T$, and $S T=T S$, then

$$
\|S+T\|^{2} \leq\|S\|^{2}+\|T\|^{2} .
$$

Thus

$$
\left\|\frac{M I-\left(P-P^{*}\right)}{1+M}\right\|^{2}=\frac{M^{2}+\left\|P-P^{*}\right\|^{2}}{M^{2}+2 M+1}
$$

which is less than 1 provided $\left\|P-P^{*}\right\|^{2}<2 M+1$, i.e., $M>\frac{1}{2}\left(\left\|P-P^{*}\right\|^{2}-1\right)$. In particular, if $M>\frac{1}{2}\left(c_{0}^{2}-1\right)$, we see that

$$
Q=P\left\{\frac{1}{M+1}\left[I-\frac{M I-\left(P-P^{*}\right)}{1+M}\right]^{-1}\right\}
$$

may be expanded in a Neumann series to give (2.5). 
We obtain, as an immediate consequence, the following:

Corollary 2.2.1. With $\left\langle P_{n}\right\rangle$ and $\left\langle Q_{n}\right\rangle$ as in the foregoing discussion, let $B$ be a real Banach function space on $(X, d \mu)$ such that $\left\langle P_{n}\right\rangle$ is uniformly holomorphic in a neighborhood of 0 in $\mathbf{B}$. Then $\left\langle Q_{n}\right\rangle$ is uniformly holomorphic in a neighborhood of 0 in $\mathbf{B}$.

The mappings $P_{n}$ may be extended to complex-valued functions in a straightforward way: if $\beta=a+i b$ is complex valued, we define, for $n \in \mathbf{N}$,

$$
P_{n}(\beta)=P_{n}(a+i b)=M_{e^{i b}} P_{n}(a) M_{e^{-i h}} .
$$

If $P_{n}(\beta)$ is a bounded operator on $L(0)$ for all $\beta$ in a neighborhood of 0 in B , then the series (2.5) may be used to extend $Q_{n}$ to complex-valued functions in a natural way, such that (by Proposition 2.2(b))

$$
Q_{n}(\beta)=P_{n}(\beta)\left[I+\left(P_{n}(\beta)-P_{n}(-\beta)\right)\right]^{-1} .
$$

Now let us compute the first Gâteaux differential at 0 , in the direction $\beta \in \mathbf{B}$, of $P_{n}$ and $Q_{n}$. We have

$$
\begin{aligned}
\left.\frac{d}{d s} P_{n}(s \beta)\right|_{s=0} & =\left.\frac{d}{d s}\left\{M_{e^{s \beta}} S_{n}(0) M_{e^{-s \beta}}\right\}\right|_{s=0} \\
& =\left.\left\{M_{\beta} M_{e^{s \beta}} S_{n}(0) M_{e^{-s \beta}}-M_{e^{s \beta}} S_{n}(0) M_{\beta} M_{e^{-s \beta}}\right\}\right|_{s=0} \\
& =\left[M_{\beta}, S_{n}(0)\right] .
\end{aligned}
$$

Thus, by (2.12), we have

$$
\text { 4) } \begin{aligned}
\left.\frac{d}{d s} Q_{n}(s \beta)\right|_{s=0} & =\left.\frac{d}{d s} P_{n}(s \beta)\right|_{s=0}-\left.P_{n}(0) \frac{d}{d s}\left\{P_{n}(s \beta)-P_{n}(-s \beta)\right\}\right|_{s=0} \\
& =\left[M_{\beta}, S_{n}(0)\right]-S_{n}\left\{\left[M_{\beta}, S_{n}(0)\right]+\left[M_{\beta}, S_{n}(0)\right]\right\} \\
& =\left\{I-2 S_{n}(0)\right\}\left[M_{\beta}, S_{n}(0)\right] .
\end{aligned}
$$

In light of these calculations we obtain

Corollary 2.2.2. Let $\mathbf{B}$ be the complexification of a real Banach function space $B$ on $(X, d \mu)$, and suppose that $\left\langle P_{n}\right\rangle$ is uniformly holomorphic in a neighborhood of 0 in $\mathbf{B}$. Then:

(a) $\mathbf{B}$ is the space of uniform holomorphy at 0 for $\left\langle P_{n}\right\rangle$ if and only if $\beta \in \mathbf{B}$ is a necessary and sufficient condition for

$$
\sup \left\{\left\|\left[M_{\beta}, S_{n}(0)\right]\right\|_{\mathscr{L}(0)}: n \in \mathbf{N}\right\}<\infty .
$$

(b) $\mathbf{B}$ is the space of uniform holomorphy at 0 for $\left\langle Q_{n}\right\rangle$ if and only if $\beta \in \mathbf{B}$ is a necessary and sufficient condition for

$$
\sup \left\{\left\|\left\{I-2 S_{n}(0)\right\}\left[M_{\beta}, S_{n}(0)\right]\right\|_{\mathscr{L}(0)}: n \in \mathbf{N}\right\}<\infty .
$$

In practice it is frequently the case that the base projections $\left\langle S_{n}(0)\right\rangle$ are given by integration against a kernel. In this case, the uniform holomorphy of $\left\langle P_{n}\right\rangle$ 
in a neighborhood of 0 may be reduced to the problem of obtaining a uniform weighted norm inequality for the base projections. This is a consequence of the following general result:

Proposition 2.3. Let $B$ be a real Banach function space on $(X, d \mu)$. Let $\left\langle K_{n}(0): n \in \mathbf{N}\right\rangle$ be a family of integral operators in $\mathscr{L}(0)$, and suppose that, for all $n \in \mathbf{N}$, there exists a kernel $D_{n}(x, y)$ such that, for $f \in L(0)$ and $x \in X$,

$$
\left\{K_{n}(0) f\right\}(x)=\int_{X} D_{n}(x, y) f(y) d \mu(y) .
$$

For each $\beta \in \mathbf{B}$, define $K_{n}(\beta)=M_{e^{\beta}} K_{n}(0) M_{e^{-\beta}}$. Then the following are equivalent:

(a) $\left\langle K_{n}\right\rangle$ is uniformly holomorphic in a neighborhood of 0 in $\mathbf{B}$.

(b) There exist constants $\delta_{0}, C_{0}>0$ such that, for every $n \in \mathbf{N}$ and for all $\beta \in B$ with $\|\beta\|_{B}<\delta_{0}$,

$$
\left\|K_{n}(\beta)\right\|_{\mathscr{L}(0)} \leq C_{0} .
$$

Proof. That (a) implies (b) is evident from Proposition 2.1. Now suppose (b) is true. By virtue of the fact that, for all $\alpha \in B$, the operator of multiplication by $e^{i \alpha}$ is an isometry of $L(0)$, it is clear that (b) continues to hold with ' $B$ ' replaced everywhere by ' $B$ '. Thus, by Proposition 2.1 , it suffices to show that each $K_{n}$ is Gâteaux differentiable in a common neighborhood of 0 in B. Our proof follows an idea of Coifman, Rochberg, and Weiss (see [3, §2]; see also [5, Chapter 4, Note 7.12]).

For $\beta \in \mathbf{B}, f \in L(0)$, and $x \in X$ we have

$$
\left\{K_{n}(\beta) f\right\}(x)=\int_{X} \exp (\beta(x)-\beta(y)) D_{n}(x, y) f(y) d \mu(y) .
$$

If $\alpha \in \mathbf{B}$, then the first Gâteaux differential of $K_{n}$ at $\alpha$ in the direction $\beta$ is given by

$$
\begin{aligned}
& \left\{\frac{d}{d z} K_{n}(\alpha+z \beta) f\right\}(x) \\
& =\int_{X}(\beta(x)-\beta(y)) \exp \{\alpha(x)-\alpha(y) \\
& +z(\beta(x)-\beta(y))\} D_{n}(x, y) f(y) d \mu(y) .
\end{aligned}
$$

Now let $\alpha, \beta \in \mathbf{B}$ with $\|\alpha\|_{\mathbf{B}}<\delta_{0} / 2$ and $\|\beta\|_{\mathbf{B}}<\left(\delta_{0} / 2\right)-\|\alpha\|_{\mathbf{B}}$. For $\theta \in$ $[0,2 \pi]$, define the operator

$$
K_{n, \theta}=K_{n}\left(\alpha+\left(z+e^{i \theta}\right) \beta\right) .
$$

Now we have

$$
\begin{aligned}
\left\|\alpha+\left(z+e^{i \theta}\right) \beta\right\|_{\mathbf{B}} & \leq\|\alpha\|_{\mathbf{B}}+(1+|z|)\|\beta\|_{\mathbf{B}} \\
& <\|\alpha\|_{\mathbf{B}}+(1+|z|)\left\{\left(\delta_{0} / 2\right)-\|\alpha\|_{\mathbf{B}}\right\} \\
& =(1+|z|)\left(\delta_{0} / 2\right)-|z|\|\alpha\|_{\mathbf{B}}
\end{aligned}
$$


which is less than $\delta_{0}$ provided $|z|<1$. Consequently, for $|z|<1$, we have $\left\|K_{n, \theta}\right\|_{\mathscr{L}(0)} \leq C_{0}$.

Now we claim that, for $|z|<1$,

$$
\frac{d}{d z} K_{n}(\alpha+z \beta)=\frac{1}{2 \pi} \int_{0}^{2 \pi} K_{n, \theta} e^{-i \theta} d \theta .
$$

In view of $(2.19)-(2.21)$, we see that, to establish (2.23), it suffices to show that

$$
\frac{1}{2 \pi} \int_{0}^{2 \pi} \exp \left\{e^{i \theta}(\beta(x)-\beta(y))\right\} e^{-i \theta} d \theta=\beta(x)-\beta(y) .
$$

But note that, if $A$ is a complex constant,

$$
\frac{1}{2 \pi} \int_{0}^{2 \pi} \exp \left(A e^{i \theta}\right) e^{-i \theta} d \theta=\frac{1}{2 \pi} \sum_{k=0}^{\infty} \int_{0}^{2 \pi} \frac{\left(A e^{i \theta}\right)^{k}}{k !} e^{-i \theta} d \theta=A .
$$

Letting $A=\beta(x)-\beta(y)$ in (2.25), we obtain (2.24).

From (2.23), we see that, for $|z|<1$,

$$
\left\|\frac{d}{d z} K_{n}(\alpha+z \beta)\right\|_{\mathscr{L}(0)} \leq \frac{1}{2 \pi} \int_{0}^{2 \pi}\left\|K_{n, \theta}\right\|_{\mathscr{L}(0)} d \theta \leq C_{0} .
$$

From this we conclude that each $K_{n}$ is Gâteaux differentiable on the open ball of radius $\delta_{0} / 2$ in $\mathbf{B}$.

With an additional assumption regarding the strong convergence of the operators $\left\langle K_{n}(\beta)\right\rangle$, we obtain the following useful result:

Proposition 2.4. Under the hypotheses of Proposition 2.3, let us make the additional assumption that there is an operator $K_{\infty}(0) \in \mathscr{L}(0)$ such that, for all $\beta$ in a neighborhood of 0 in $B, K_{n}(\beta)-K_{\infty}(\beta)$ converges to 0 in the strong operator topology on $\mathscr{L}(0)$ as $n \rightarrow \infty$, where $K_{\infty}(\beta)=M_{e^{\beta}} K_{\infty}(0) M_{e^{-\beta}}$. Then the following are equivalent:

(a) There exist constants $\delta_{0}, C_{0}>0$ such that, for all $n \in \mathbf{N}$ and for all $\beta \in B$ with $\|\beta\|_{B}<\delta_{0}$, inequality (2.18) holds.

(b) There exist constants $\delta_{1}, C_{1}>0$ such that, for all $\beta \in B$ with $\|\beta\|_{B}<\delta_{1}$,

$$
\left\|K_{\infty}(\beta)\right\|_{\mathscr{L}(0)} \leq C_{1} .
$$

(c) $\left\langle K_{n}\right\rangle$ is uniformly holomorphic in a neighborhood of 0 in $\mathbf{B}$.

Proof. The equivalence of (a) and (c) is simply Proposition 2.3. The equivalence of $(a)$ and $(b)$ is the essence of a remark made by Garnett [6, p. 109]. We give the details.

Suppose that (a) holds. Let $\varepsilon>0$ and $\beta \in B$ with $\|\beta\|_{B}<\delta_{0}$. For every $f \in L(0)$ we can find $N>0$ such that $n \geq N$ implies

$$
\left\|\left\{K_{n}(\beta)-K_{\infty}(\beta)\right\} f\right\|_{L(0)}<\varepsilon .
$$

Then, for such $n$, we have

$$
\begin{aligned}
\left\|K_{\infty}(\beta) f\right\|_{L(0)} & \leq\left\|\left\{K_{n}(\beta)-K_{\infty}(\beta)\right\} f\right\|_{L(0)}+\left\|K_{n}(\beta) f\right\|_{L(0)} \\
& <\varepsilon+C_{0}\|f\|_{L(0)} .
\end{aligned}
$$


Since $\varepsilon$ was arbitrary, we see that (b) is true with $\delta_{1}=\delta_{0}$ and $C_{1}=C_{0}$.

Conversely, assume that (b) is true. Let $\varepsilon>0$ and $\beta \in B$ with $\|\beta\|_{B}<\delta_{1}$. For every $f \in L(0)$ we can find $N>0$ such that $n \geq N$ implies (2.28). For such $n$, we have

$$
\begin{aligned}
\left\|K_{n}(\beta) f\right\|_{L(0)} & \leq\left\|\left\{K_{n}(\beta)-K_{\infty}(\beta)\right\} f\right\|_{L(0)}+\left\|K_{\infty}(\beta) f\right\|_{L(0)} \\
& <\varepsilon+C_{1}\|f\|_{L(0)} .
\end{aligned}
$$

Thus the family of operators $\left\{K_{n}(\beta): n \in \mathbf{N}, \beta \in B,\|\beta\|_{B}<\delta_{1}\right\}$ is "pointwise bounded" on $L(0)$. Then (a) follows from the principle of uniform boundedness.

\section{UNIFORM ANALYTICITY ON THE CIRCLE}

In this section we apply our work in $\S 2$ to the case of trigonometric polynomials on the circle, $\mathbf{T}$. We parametrize $\mathbf{T}$ by the interval $[-\pi, \pi)$, and let $L(0)=L^{2}(\mathbf{T})=L^{2}([-\pi, \pi), d \theta)$, where $d \theta$ is ordinary Lebesgue measure. Let $\omega$ be a nonnegative weight function on $\mathbf{T}$ such that $\omega^{2}+\omega^{-2} \in L^{1}(\mathbf{T})$, and write $\beta=\log \omega$. For each integer $k$, define the function $e_{k}$ by $e_{k}(\theta)=e^{i k \theta}$. We define, for $n \in \mathbf{N}$, the space

$$
H_{n}(0)=\operatorname{span}_{\mathbf{C}}\left\langle e_{k}:|k| \leq n\right\rangle
$$

of trigonometric polynomials of degree at most $n$; we define $L(\beta), \mathscr{L}(\beta)$, $H_{n}(\beta)$, etc. as in $\S 2$. We note that the base projections $\left\langle S_{n}(0): n \in \mathbf{N}\right\rangle$ are simply the partial sum operators for Fourier series, defined by

$$
S_{n}(0) f=\sum_{k=-n}^{n} \hat{f}(k) e_{k}
$$

where, for each integer $k, \hat{f}(k)$ is the $k$ th Fourier coefficient of $f$, given by

$$
\hat{f}(k)=\frac{1}{2 \pi} \int_{-\pi}^{\pi} f(\theta) e_{-k}(\theta) d \theta
$$

and thus

$$
\sum_{k=-\infty}^{\infty} \hat{f}(k) e_{k}
$$

is the Fourier series for $f$. The operator $S_{n}(0)$ is an integral operator, given by

$$
\left\{S_{n}(0) f\right\}(\theta)=\frac{1}{2 \pi} \int_{-\pi}^{\pi} D_{n}(\theta, \psi) f(\psi) d \psi
$$

where $D_{n}(\theta, \psi)$ is the Dirichlet kernel, given by

$$
D_{n}(\theta, \psi)=\sum_{k=-n}^{n} e_{k}(\theta) e_{-k}(\psi)=\frac{\sin \left[(2 n+1) \frac{\theta-\psi}{2}\right]}{\sin \left(\frac{\theta-\psi}{2}\right)}
$$


(see, for example, [10, p. 12]).

We shall show that, in this example, the space of functions of bounded mean oscillation on $\mathbf{T}$ is the space of uniform holomorphy at 0 for the families $\left\langle P_{n}\right\rangle$ and $\left\langle Q_{n}\right\rangle$.

We adopt the convention that $I$ is a subinterval of $\mathbf{T}$ if and only if it is a subinterval of $[-\pi, \pi)$ in the usual sense, or it is the union of an interval of the form $(c, \pi)$ or $[c, \pi)$ with an interval of the form $[-\pi, d)$ or $[-\pi, d]$, with $-\pi<d<c<\pi$. We let $|I|$ denote the Lebesgue measure of $I$. If $b \in L^{1}(\mathbf{T})=L^{1}([-\pi, \pi), d \theta)$, we define the mean of $b$ on $I$ to be

$$
m_{I}(b)=|I|^{-1} \int_{I} b(\theta) d \theta .
$$

The function $b$ is said to have bounded mean oscillation on $\mathbf{T}$ if and only if the quantity

$$
\|b\|_{*} \equiv \sup _{I}|I|^{-1} \int_{I}\left|b(\theta)-m_{I}(b)\right| d \theta=\sup _{I} m_{I}\left(\left|b-m_{I}(b)\right|\right)
$$

is finite, where the supremum is taken over all subintervals $I$ of $\mathbf{T}$. The space $B M O(\mathrm{~T})$ of real-valued functions (modulo constants) having bounded mean oscillation on $\mathbf{T}$ is a Banach space with $\|\cdot\|_{*}$ as its norm. For ease of notation in this section we shall refer to $B M O(\mathrm{~T})$ as simply $B M O$; its complexification will be denoted by BMO.

To begin, we shall show that $\left\langle P_{n}\right\rangle$ is uniformly holomorphic in a neighborhood of 0 in BMO, from which it follows that the same is true of $\left\langle Q_{n}\right\rangle$, by Corollary 2.2.1. By Proposition 2.3, the uniform holomorphy of $\left\langle P_{n}\right\rangle$ in a neighborhood of 0 in BMO is equivalent to a uniform estimate of the form

$$
\left\|P_{n}(\beta)\right\|_{\mathscr{L}(0)} \leq C_{0} \text { for all } \beta \in B M O \text { with }\|\beta\|_{*}<\delta_{0},
$$

where $C_{0}, \delta_{0}$ are constants independent of $n$.

We can, in fact, characterize the weight functions $\omega=e^{\beta}$ for which $\left\langle\left\|P_{n}(\beta)\right\|_{\mathscr{L}(0)}\right\rangle$ is uniformly bounded in $n$. Recall that the weight $\omega$ is said to belong to the class $A_{2}$ if and only if

$$
\sup _{I} m_{I}(\omega) m_{I}\left(\omega^{-1}\right)<\infty
$$

where the supremum is taken over all subintervals $I$ of $\mathbf{T}$. The quantity (3.10) is called the $A_{2}$ constant of $\omega$. We have the following result:

Proposition 3.1. The quantity $\sup \left\langle\left\|P_{n}(\beta)\right\|_{\mathscr{L}(0)}: n \in \mathbf{N}\right\rangle$ is finite if and only if $\omega^{2} \in A_{2}$.

Proof. The proof is analogous to that of [5, Corollary 3.12, Chapter 4]. The idea is to exploit the relationship between $S_{n}(0)$ and the orthogonal projection $P_{+}$of $L(0)$ onto the Hardy space $\mathscr{H}_{+}^{2}=\{f \in L(0): \hat{f}(k)=0$ for $k<0\}$. 
Consider the operator $T_{n}=e_{n} S_{n}(0) e_{-n}$. A simple computation shows that, for $f \in L(0)$,

$$
T_{n} f=\sum_{k=0}^{2 n} \hat{f}(k) e_{k} .
$$

Moreover, as $n \rightarrow \infty, T_{n} \rightarrow P_{+}$in the strong operator topology on $\mathscr{L}(0)$. By a slight modification of the proof of Proposition 2.4, it follows that $\sup \left\langle\left\|M_{\omega} T_{n} M_{\omega}^{-1}\right\|_{\mathscr{L}(0)}: n \in \mathrm{N}\right\rangle$ is finite if and only if $M_{\omega} P_{+} M_{\omega}^{-1} \in \mathscr{L}(0)$. By virtue of the relationship between $P_{+}$and the conjugate operator (cf. $[6, \mathrm{p}$. 108]), we see that $M_{\omega} P_{+} M_{\omega}^{-1} \in \mathscr{L}(0)$ if and only if $\omega^{2} \in A_{2}$ (see [7]). Now note that

$$
P_{n}(\beta)=M_{e_{-n}} M_{\omega} T_{n} M_{\omega}^{-1} M_{e_{n}} ;
$$

moreover, $M_{e_{k}}$ is an isometry for each integer $k$. Consequently, for each integer $n$,

$$
\left\|P_{n}(\beta)\right\|_{\mathscr{L}(0)}=\left\|M_{\omega} T_{n} M_{\omega}^{-1}\right\|_{\mathscr{L}(0)},
$$

from which the result follows.

Corollary 3.1.1. $\left\langle P_{n}\right\rangle$ and $\left\langle Q_{n}\right\rangle$ are uniformly holomorphic in a neighborhood of 0 in BMO.

Proof. There exist constants $\delta_{0}, C>0$ such that, if $\beta \in B M O$ and $\|\beta\|_{*}<$ $\delta_{0}$, then $\omega^{2} \in A_{2}$, and the $A_{2}$ constant of $\omega^{2}$ is less than or equal to $C$ (see [5, Chapter 2, Corollary 3.10 and Chapter 4, Corollary 2.18]). For $\omega^{2} \in A_{2}$, the $\mathscr{L}(0)$-norm of $M_{\omega} P_{+} M_{\omega}^{-1}$ depends upon the $A_{2}$ constant of $\omega^{2}$; so by the proof of Proposition 3.1, we obtain (3.9), from which the corollary follows.

Next, we would like to show that BMO is actually the space of uniform holomorphy at 0 for $\left\langle P_{n}\right\rangle$ and $\left\langle Q_{n}\right\rangle$. A simple computation shows that $I-$ $2 S_{n}(0)$ is an isometry of $L(0)$ for each $n \in \mathbf{N}$; so, by Corollary 2.2.2, it suffices to show that $\beta \in \mathbf{B M O}$ is a necessary and sufficient condition for boundedness of the set $\left\{\left\|\left[M_{\beta}, S_{n}(0)\right]\right\|_{\mathscr{L}(0)}: n \in \mathbf{N}\right\}$. We have the following:

Proposition 3.2. There exist constants $C_{1}, C_{2}>0$ such that for all $\beta \in L^{1}(\mathbf{T})$,

$$
C_{1}\|\beta\|_{*} \leq \sup _{n}\left\|\left[M_{n}, S_{n}(0)\right]\right\|_{\mathscr{L}(0)} \leq C_{2}\|\beta\|_{*} .
$$

Proof. The existence of $C_{2}$ with the desired property follows from the fact that $\left\langle P_{n}\right\rangle$ is uniformly holomorphic in a neighborhood of 0 in BMO. Therefore it suffices to prove that there exists a constant $\mu>0$ such that, for all $\beta \leq L^{1}(\mathbf{T})$,

$$
\|\beta\|_{*} \leq \mu \sup _{n}\left\|\left[M_{\beta}, S_{n}(0)\right]\right\|_{\mathscr{L}(0)} .
$$

Let $I$ be a subinterval of $T$ and let $\theta \in[-\pi, \pi)$. Define the function $f_{\theta}=f_{I, \theta, n}$ by setting, for $\psi \in[-\pi, \pi)$,

$$
f_{\theta}(\psi)=2 i \sin \left(\frac{\theta-\psi}{2}\right) \exp \left[i(2 n+1) \frac{\theta-\psi}{2}\right] \chi_{I}(\psi) .
$$


Observe that, by (3.6), when $\psi \in I$,

$$
\begin{aligned}
D_{n}(\theta, \psi) f_{\theta}(\psi)= & 2 i \sin \left[(2 n+1) \frac{\theta-\psi}{2}\right] \exp \left[i(2 n+1) \frac{\theta-\psi}{2}\right] \chi_{I}(\psi) \\
= & 2 i \cos \left[(2 n+1) \frac{\theta-\psi}{2}\right] \sin \left[(2 n+1) \frac{\theta-\psi}{2}\right] \chi_{I}(\psi) \\
& -2 \sin ^{2}\left[(2 n+1) \frac{\theta-\psi}{2}\right] \chi_{I}(\psi) .
\end{aligned}
$$

In view of the fact that

$$
\begin{aligned}
& \exp [i(2 n+1)(\theta-\psi)] \\
&=1-2 \sin ^{2}\left[(2 n+1) \frac{\theta-\psi}{2}\right] \\
&+2 i \cos \left[(2 n+1) \frac{\theta-\psi}{2}\right] \sin \left[(2 n+1) \frac{\theta-\psi}{2}\right],
\end{aligned}
$$

we obtain, for $\psi \in I$,

$$
D_{n}(\theta, \psi) f_{\theta}(\psi)=(\exp [i(2 n+1)(\theta-\psi)]-1) \chi_{I}(\psi) .
$$

Consequently, by (3.5) and (3.19),

(3.20) $\left\{\left[M_{\beta}, S_{n}(0)\right] f_{\theta}\right\}(\theta)$

$$
\begin{aligned}
= & \frac{1}{2 \pi} \int_{-\pi}^{\pi}\{\exp i(2 n+1)(\theta-\psi)-1\}(\beta(\theta)-\beta(\psi)) \chi_{I}(\psi) d \psi \\
= & \frac{1}{2 \pi} \int_{-\pi}^{\pi} \exp [i(2 n+1)(\theta-\psi)](\beta(\theta)-\beta(\psi)) \chi_{I}(\psi) d \psi \\
& -\frac{1}{2 \pi} \int_{-\pi}^{\pi}(\beta(\theta)-\beta(\psi)) \chi_{I}(\psi) d \psi \\
= & \frac{1}{2 \pi} \int_{-\pi}^{\pi} \exp [i(2 n+1)(\theta-\psi)](\beta(\theta)-\beta(\psi)) \chi_{I}(\psi) d \psi \\
& -\frac{1}{2 \pi}|I|\left(\beta(\theta)-m_{I}(\beta)\right) .
\end{aligned}
$$

Now note that

$$
\text { (3.21) } \begin{aligned}
\frac{1}{2 \pi} \int_{-\pi}^{\pi} \exp [i(2 n+1)(\theta-\psi)](\beta(\theta)-\beta(\psi)) \chi_{I}(\psi) d \psi \\
=\exp [i(2 n+1) \theta]\left\{\beta(\theta) \cdot \frac{1}{2 \pi} \int_{-\pi}^{\pi} \exp [-i(2 n+1) \psi] \chi_{I}(\psi) d \psi\right. \\
\left.\quad-\frac{1}{2 \pi} \int_{-\pi}^{\pi} \exp [-i(2 n+1) \psi] \beta(\psi) \chi_{I}(\psi) d \psi\right\} \\
=\exp [i(2 n+1) \theta]\left\{\beta(\theta) \hat{\chi}_{I}(2 n+1)-\widehat{\beta \chi}_{i}(2 n+1)\right\} .
\end{aligned}
$$

Consequently, by (3.20) and (3.21),

(3.22) $\left\{\left[M_{\beta}, S_{n}(0)\right] f_{\theta}\right\}(\theta)=\exp [i(2 n+1) \theta]\left\{\beta(\theta) \hat{\chi}_{I}(2 n+1)-\widehat{\beta \chi}_{I}(2 n+1)\right\}$

$$
-\frac{1}{2 \pi}|I|\left(\beta(\theta)-m_{I}(\beta)\right) \text {. }
$$


Letting

$$
\begin{gathered}
g_{n}(\theta)=\chi_{I}(\theta) \exp [i(2 n+1) \theta]\left\{\beta(\theta) \hat{\chi}_{I}(2 n+1)-\widehat{\beta \chi}_{I}(2 n+1)\right\}, \\
g(\theta)=\frac{1}{2 \pi}|I|\left(\beta(\theta)-m_{I}(\beta)\right),
\end{gathered}
$$

we see that

$$
\int_{I}\left|\left\{\left[M_{\beta}, S_{n}(0)\right] f_{\theta}\right\}(\theta)\right| d \theta=\left\|g-g_{n}\right\|_{1}
$$

Now

$$
\left\|g_{n}\right\|_{1} \leq\left|\hat{\chi}_{I}(2 n+1)\right|\|\beta\|_{1}+\left|\widehat{\beta \chi}_{I}(2 n+1)\right||I| ;
$$

the right-hand side of (3.26) tends to 0 as $n \rightarrow \infty$, by the Riemann-Lebesgue lemma, so $\lim _{n \rightarrow \infty}\left\|g_{n}\right\|_{1}=0$. Consequently, by Fatou's lemma,

$$
\lim _{n \rightarrow \infty} \int_{I}\left|\left\{\left[M_{\beta}, S_{n}(0)\right] f_{\theta}\right\}(\theta)\right| d \theta=\|g\|_{1}=\frac{1}{2 \pi}|I| \int_{I}\left|\beta(\theta)-m_{I}(\beta)\right| d \theta .
$$

Thus (3.15) follows, once we prove an estimate of the form

$$
\varlimsup_{n \rightarrow \infty} \int_{I}\left|\left\{\left[M_{\beta}, S_{n}(0)\right] f_{\theta}\right\}(\theta)\right| d \theta \leq \frac{1}{2 \pi} \mu C|I|^{2}
$$

where

$$
C=\sup _{n}\left\|\left[M_{\beta}, S_{n}(0)\right]\right\|_{\mathscr{L}(0)}
$$

and $\mu$ is a constant independent of $\beta$ and $I$.

Now we have

$$
\begin{aligned}
& \left\{\left[M_{\beta}, S_{n}(0)\right] f_{\theta}\right\}(\theta) \\
& \begin{aligned}
=\frac{1}{2 \pi} \int_{-\pi}^{\pi} D_{n}(\theta, \psi)(\beta(\theta)-\beta(\psi)) f_{\theta}(\psi) d \psi & \\
=\frac{1}{2 \pi} \int_{-\pi}^{\pi} D_{n}(\theta, \psi)(\beta(\theta)-\beta(\psi)) 2 i \sin \left(\frac{\theta-\psi}{2}\right) & \quad \times \exp \left[i(2 n+1) \frac{\theta-\psi}{2}\right] \chi_{I}(\psi) d \psi \\
=2 i \exp \left[i(2 n+1) \frac{\theta}{2}\right] \cdot \frac{1}{2 \pi} \int_{-\pi}^{\pi} D_{n}(\theta, \psi)(\beta(\theta)-\beta(\psi)) \sin \left(\frac{\theta-\psi}{2}\right) & \quad \times \exp \left[-i(2 n+1) \frac{\psi}{2}\right] \chi_{I}(\psi) d \psi
\end{aligned}
\end{aligned}
$$

so that

(3.31) $\left|\left\{\left[M_{\beta}, S_{n}(0)\right] f_{\theta}\right\}(\theta)\right|$

$$
\begin{aligned}
=2 \cdot \mid \frac{1}{2 \pi} \int_{-\pi}^{\pi} D_{n}(\theta, \psi)(\beta(\theta) & -\beta(\psi)) \sin \left(\frac{\theta-\psi}{2}\right) \\
& \times \exp \left[-i(2 n+1) \frac{\psi}{2}\right] \chi_{I}(\psi) d \psi \mid .
\end{aligned}
$$


Now suppose $I$ is a subinterval of $\mathbf{T}$ which is also a subinterval of $[-\pi, \pi)$ in the ordinary sense, and let $\psi_{0}$ denote the ordinary midpoint of $I$. (If $I$ is a subinterval of $\mathbf{T}$ comprising two disjoint subintervals of $[-\pi, \pi)$, we need to make minor adjustments to the argument.) Now, we write

$$
\begin{aligned}
\sin \left(\frac{\theta-\psi}{2}\right) & =\sin \left(\frac{\theta-\psi_{0}+\psi_{0}-\psi}{2}\right) \\
& =\sin \left(\frac{\theta-\psi_{0}}{2}\right) \cos \left(\frac{\psi_{0}-\psi}{2}\right)+\cos \left(\frac{\theta-\psi_{0}}{2}\right) \sin \left(\frac{\psi_{0}-\psi}{2}\right),
\end{aligned}
$$

so that

$$
\begin{aligned}
\left|\left\{\left[M_{\beta}, S_{n}(0)\right] f_{\theta}\right\}(\theta)\right| \leq & 2\left|\sin \left(\frac{\theta-\psi_{0}}{2}\right)\left\{\left[M_{\beta}, S_{n}(0)\right] h_{1}\right\}(\theta)\right| \\
& +2\left|\cos \left(\frac{\theta-\psi_{0}}{2}\right)\left\{\left[M_{\beta}, S_{n}(0)\right] h_{2}\right\}(\theta)\right|
\end{aligned}
$$

where

$$
\begin{aligned}
& h_{1}(\psi)=\cos \left(\frac{\psi_{0}-\psi}{2}\right) \exp \left[-i(2 n+1) \frac{\psi}{2}\right] \chi_{I}(\psi), \\
& h_{2}(\psi)=\sin \left(\frac{\psi_{0}-\psi}{2}\right) \exp \left[-i(2 n+1) \frac{\psi}{2}\right] \chi_{I}(\psi) .
\end{aligned}
$$

Now, for $\alpha \in \mathbf{R}$, we have $|\sin \alpha| \leq|\alpha| \cosh \alpha$, so that, for $\psi, \theta \in I$,

$$
\begin{gathered}
\left|h_{2}(\psi)\right| \leq\left|\frac{\psi_{0}-\psi}{2}\right| \cosh \left(\frac{\psi_{0}-\psi}{2}\right) \leq \frac{1}{2}(\cosh \pi)\left|\psi_{0}-\psi\right| \\
\left|\sin \left(\frac{\theta-\psi_{0}}{2}\right)\right| \leq\left|\frac{\theta-\psi_{0}}{2}\right| \cosh \left(\frac{\theta-\psi_{0}}{2}\right) \leq \frac{1}{2}(\cosh \pi)\left|\theta-\psi_{0}\right|
\end{gathered}
$$

Thus, by Schwarz' inequality, (3.33) and (3.36),

$$
\begin{aligned}
\frac{1}{2} \int_{I}\left|\left\{\left[M_{\beta}, S_{n}(0)\right] f_{\theta}\right\}(\theta)\right| d \theta \\
\leq\left(\int_{I}\left|\sin \left(\frac{\theta-\psi_{0}}{2}\right)\right|^{2} d \theta\right)^{1 / 2}\left\|\left[M_{\beta}, S_{n}(0)\right] h_{1}\right\|_{2} \\
\quad+\left(\int_{I}\left|\cos \left(\frac{\theta-\psi_{0}}{2}\right)\right|^{2} d \theta\right)^{1 / 2}\left\|\left[M_{\beta}, S_{n}(0)\right] h_{2}\right\|_{2} \\
\leq \frac{1}{2}(\cosh \pi)\left(\int_{I}\left|\theta-\psi_{0}\right|^{2} d \theta\right)^{1 / 2} C\left\|h_{1}\right\|_{2}+|I|^{1 / 2} C\left\|h_{2}\right\|_{2} \\
=\frac{\sqrt{3}}{12}(\cosh \pi)|I|^{3 / 2} C\left\|h_{1}\right\|_{2}+|I|^{1 / 2} C\left\|h_{2}\right\|_{2} .
\end{aligned}
$$

We have

$$
\left\|h_{1}\right\|_{2}=\left(\int_{I}\left|\cos \left(\frac{\psi-\psi_{0}}{2}\right)\right|^{2} d \psi\right)^{1 / 2} \leq|I|^{1 / 2}
$$




$$
\begin{aligned}
\left\|h_{2}\right\| & =\left(\int_{I}\left|\sin \left(\frac{\psi-\psi_{0}}{2}\right)\right|^{2} d \psi\right)^{1 / 2} \\
& \leq \frac{1}{2}(\cosh \pi)\left(\int_{I}\left|\psi_{0}-\psi\right|^{2} d \psi\right)^{1 / 2} \\
& =\frac{\sqrt{3}}{12}(\cosh \pi)|I|^{3 / 2}
\end{aligned}
$$

so that

$$
\int_{I}\left|\left\{\left[M_{\beta}, S_{n}(0)\right] f_{\theta}\right\}(\theta)\right| d \theta \leq \frac{\sqrt{3}}{3}(\cosh \pi) C|I|^{2}
$$

whence

$$
\varlimsup_{n \rightarrow \infty} \int_{I}\left|\left\{\left[M_{\beta}, S_{n}(0)\right] f_{\theta}\right\}(\theta)\right| d \theta \leq \frac{1}{2 \pi}\left[\frac{2 \pi \sqrt{3} \cosh \pi}{3}\right] C|I|^{2},
$$

which is (3.28), with $\mu=(2 \pi \sqrt{3} \cosh \pi) / 3$. This completes the proof.

The following corollary is immediate.

Corollary 3.2.1. BMO is the space of uniform holomorphy at 0 for $\left\langle P_{n}\right\rangle$ and $\left\langle Q_{n}\right\rangle$.

\section{UNIFORM ANALYTICITY ON $[-1,1]$ IN A NEIGHBORHOOD OF A JACOBI WEIGHT}

In this section we consider an example on the interval $[-1,1]$. For $\gamma, \delta>$ -1 , we define the Jacobi weight with parameters $\gamma, \delta$, by

$$
\omega_{\gamma, \delta}^{2}(x)=(1-x)^{\gamma}(1+x)^{\delta} .
$$

Our basic Hilbert space is

$$
L_{\gamma, \delta}(0)=L^{2}\left([-1,1], \omega_{\gamma, \delta}^{2}(x) d x\right),
$$

and we shall denote the orthogonal projection onto polynomials of degree $\leq \boldsymbol{n}$ in $L_{\gamma, \delta}(0)$ by $S_{n}^{\gamma, \delta}(0)$. We shall make a small multiplicative perturbation of $\omega_{\gamma, \delta}^{2}(x) d x$ by a nonnegative weight function $\omega^{2}=e^{2 \beta}$, where

$$
\omega^{2}+\omega^{-2} \in L^{1}\left([-1,1], \omega_{\gamma, \delta}^{2}(x) d x\right), \quad \beta \in L^{1}([-1,1], d x) .
$$

The corresponding Hilbert space is

$$
L_{\gamma, \delta}(\beta)=L^{2}\left([-1,1], \omega_{\gamma, \delta}^{2}(x) \omega^{2}(x) d x\right) .
$$

This orthogonal projection onto polynomials of degree $\leq n$ in $L_{\gamma, \delta}(\beta)$ will be denoted by $S_{n}^{\gamma, \delta}(\beta)$. Setting

$$
\begin{aligned}
& Q_{n}^{\gamma, \delta}(\beta)=M_{\omega} S_{n}^{\gamma, \delta}(\beta) M_{\omega}^{-1}, \\
& P_{n}^{\gamma, \delta}(\beta)=M_{\omega} S_{n}^{\gamma, \delta}(0) M_{\omega}^{-1},
\end{aligned}
$$


we conjecture that the space of functions of bounded mean oscillation on $[-1,1]$ is the space of uniform holomorphy at 0 for the families $\left\langle Q_{n}^{\gamma, \delta}\right\rangle$ and $\left\langle P_{n}^{\gamma, \delta}\right\rangle$ whenever $\gamma, \delta>-1$. In fact, we shall show that $\left\langle Q_{n}^{\gamma, \delta}\right\rangle$ and $\left\langle P_{n}^{\gamma, \delta}\right\rangle$ are uniformly holomorphic in a neighborhood of 0 in $\mathbf{B M O}([-1,1])$ whenever $\gamma, \delta \geq-\frac{1}{2}$.

We pause to remark that the operator $S_{n}^{\gamma, \delta}(0)$ is the $n$th partial sum operator for Jacobi series with parameters $\gamma, \delta$; it is an integral operator whose kernel may be expressed in terms of the Jacobi polynomials $\left\langle P_{n}^{(\gamma, \delta)}(x): n \in \mathbf{N}\right\rangle$, which form an orthogonal polynomial system in $L_{\gamma, \delta}(0)$ (see [10, Chapters 3 and 4]; see also [9]). Making use of Christoffel-Darboux formulas for $S_{n}^{\gamma, \delta}(0)$, it is possible to reduce the problem of obtaining uniform weighted norm inequalities for $S_{n}^{\gamma, \delta}(0)$ to that of obtaining weighted norm inequalities for the Hilbert transform, as we shall see.

We begin with some terminology. A function $b \in L^{1}([-1,1], d x)$ is in $\operatorname{BMO}([-1,1], d x)$ if and only if

$$
\|b\|_{*} \equiv \sup _{I}|I|^{-1} \int_{I}\left|b(\theta)-m_{I}(b)\right| d \theta
$$

is finite, where the supremum is taken over all subintervals $I$ of $[-1,1]$. $\operatorname{BMO}([-1,1], d x)$ is a Banach space of functions (modulo constants). The space of real-valued functions in $\mathbf{B M O}([-1,1]), d x)$ will be denoted by $B M O([-1,1], d x)$; we will use the abbreviations BMO and BMO. A nonnegative weight function $\omega$ belongs to the class $A_{2}$ if and only if

$$
\sup _{I} m_{I}(\omega) m_{I}\left(\omega^{-1}\right)<\infty
$$

where the supremum is taken over all subintervals $I$ of $[-1,1]$; the quantity (4.8) is called the $A_{2}$ constant of $\omega$.

By analogy to the notation of $\S 2$, we let $\mathscr{L}_{\gamma, \delta}(0)$ denote the Hilbert space of bounded linear operators on $L_{\gamma, \delta}(0)$. We wish to obtain a uniform estimate of the form

$$
\left\|P_{n}^{\gamma, \delta}(\beta)\right\|_{\mathscr{L}_{i, \delta}(0)} \leq C
$$

which is valid for all $\beta$ in some neighborhood of the origin in $B M O$. Note that the estimate (4.9) is equivalent to an estimate of form

$$
\left\|M_{\omega} M_{\omega_{i, \delta}} S_{n}^{\gamma, \delta}(0) M_{\omega_{i, \delta}}^{-1} M_{\omega}^{-1}\right\|_{\mathscr{L}(0)} \leq C,
$$

where $\mathscr{L}(0)=\mathscr{L}_{0,0}(0)$, which is valid for all $\beta$ in some neighborhood of the origin in $B M O$. In order to prove an estimate of this form, we need the following lemma.

Lemma 4.1. Suppose $w^{2} \in A_{2}$. Then there exist constants $\delta_{1}, C>0$, depending only upon $w$, such that for all $\beta \in B M O$ with $\|\beta\|_{*}<\delta_{1}, e^{2 \beta} w^{2}$ is also in $A_{2}$ with an $A_{2}$ constant less than or equal to $C$. 
Proof. We make use of the following characterization of $A_{2}$ : a function $\varphi \in$ $L^{1}([-1,1], d x)$ is the logarithm of $A_{2}$ weight if and only if the quantity

$$
\sup _{I}|I|^{-1} \int_{I} \exp \left(\left|\varphi(x)-m_{I}(\varphi)\right|\right) d x
$$

(where the supremum is taken over all subintervals $I$ of $[-1,1]$ ) is finite; the quantity (4.11) is equivalent to the square root of the $A_{2}$ constant of $e^{\varphi}$ (see [5, Chapter 4, Theorem 2.17 and Corollary 2.18]).

Now suppose $w^{2} \in A_{2}$; let $f=\log w$. By [5, Theorem 2.7, Chapter 4] there is a constant $\varepsilon>0$ such that $w^{2+\varepsilon} \in A_{2}$. Consequently, if $I$ is a subinterval of $[-1,1]$, we have

$$
\begin{aligned}
& |I|^{-1} \int_{I} \exp \left(\left|(2 \beta+2 f)(x)-m_{I}(2 \beta+2 f)\right|\right) d x \\
& =|I|^{-1} \int_{I} \exp \left|2 \beta(x)-m_{I}(2 \beta)\right| \exp \left|2 f(x)-m_{I}(2 f)\right| d x \\
& \leq|I|^{-1}\left(\int_{I}\left[\exp \left|2 \beta(x)-m_{I}(2 \beta)\right|\right]^{(2+\varepsilon) / \varepsilon} d x\right)^{\varepsilon /(2+\varepsilon)} \\
& \quad \times\left(\int_{I}\left[\exp \left|2 f(x)-m_{I}(2 f)\right|\right]^{(2+\varepsilon) / 2}\right)^{2 /(2+\varepsilon)} \\
& =\left(|I|^{-1} \int_{I} \exp \left|\left(\frac{4+2 \varepsilon}{\varepsilon}\right)\left(\beta(x)-m_{I}(\beta)\right)\right| d x\right)^{\varepsilon /(2+\varepsilon)} \\
& \quad \times\left(|I|^{-1} \int_{I} \exp \left|(2+\varepsilon)\left(f(x)-m_{I}(f)\right)\right| d x\right)^{2 /(2+\varepsilon)}
\end{aligned}
$$

by Hölder's inequality. Now, there exist constants $\delta_{0}, C>0$ such that, if $\varphi \in B M O$ and $\|\varphi\|_{*}<\delta_{0}$, then $e^{2 \varphi} \in A_{2}$, with an $A_{2}$ constant less than or equal to $C$ (see [5, Chapter 2, Corollary 3.10 and Chapter 4, Corollary 2.18]). Taking $\delta_{1}=\varepsilon \delta_{0} /(2+\varepsilon)$, then $\|((2+\varepsilon) / \varepsilon) \beta\|_{*}<\delta_{0}$ when $\|\beta\|_{*}<\delta_{1}$. Consequently, if $\|\beta\|_{*}<\delta_{1}$ then (4.12) will be dominated by a constant which depends only upon $w$ and which is independent of $I$. The result follows.

We can now prove:

Proposition 4.2. Let $\gamma, \delta \geq-\frac{1}{2}$. Then there exist constants $C, \delta_{1}>0$ such that for all $n \in \mathbf{N}$ and for all $\beta \in B M O$ with $\|\beta\|_{*}<\delta_{1}$,

$$
\left\|M_{\omega} M_{\omega_{i, \delta} \delta} S_{n}^{\gamma, \delta}(0) M_{\gamma, \delta}^{-1} M_{\omega}^{-1}\right\|_{\mathscr{L}(0)} \leq C
$$

where $\omega=e^{\beta}$.

Proof. We make use of Muckenhoupt's work in [9]. First of all, we note that, for $f \in L_{\gamma, \delta}(0)$,

$$
S_{n}^{\gamma, \delta}(0) f(x)=\int_{-1}^{1} K_{n}^{\gamma, \delta}(x, y) f(y) \omega_{\gamma, \delta}^{2}(y) d y,
$$


where

$$
K_{n}^{\gamma, \delta}(x, y)=\sum_{j=0}^{n} P_{j}^{(\gamma, \delta)}(x) P_{j}^{(\gamma, \delta)}(y)\left\|P_{j}^{(\gamma, \delta)}\right\|_{L_{, j}(0)}^{-2} .
$$

Thus we have, for $f \in L(0)$,

$$
M_{\omega} M_{\omega_{\gamma, \delta}} S_{n}^{\gamma, \delta}(0) M_{\omega_{\gamma, \delta}}^{-1} M_{\omega}^{-1} f(x)=\int_{-1}^{1} K_{n}^{\gamma, \delta}(x, y) \omega_{\gamma, \delta}(x) \omega_{\gamma, \delta}(y) \frac{\omega(x)}{\omega(y)} f(y) d y .
$$

Using estimates from [10], Muckenhoupt writes

$$
\begin{aligned}
K_{n}^{\gamma, \delta}(x, y)= & A(n, \gamma, \delta) H_{1}^{\gamma, \delta}(n ; x, y) \\
& +B(n, \gamma, \delta)\left[H_{2}^{\gamma, \delta}(n ; x, y)+H_{2}^{\gamma, \delta}(n ; y, x)\right]
\end{aligned}
$$

where $|A(n, \gamma, \delta)|,|B(n, \gamma, \delta)|$ are bounded above by a constant independent of $n$, and

$$
\begin{gathered}
H_{1}^{\gamma, \delta}(n ; x, y)=(n+1) P_{n}^{(\gamma, \delta)}(x) P_{n}^{(\gamma, \delta)}(y), \\
H_{2}^{\gamma, \delta}(n ; x, y)=\frac{n\left(1-y^{2}\right) P_{n}^{(\gamma, \delta)}(x) P_{n-1}^{(\gamma+1, \delta+1)}(y)}{x-y} .
\end{gathered}
$$

The Jacobi polynomials satisfy

$$
P_{n}^{(\gamma, \delta)}(x)=(-1)^{n} P_{n}^{(\gamma, \delta)}(-x), \quad x \in[-1,1]
$$

(see $[10$, p. $59,(4.13)])$; moreover, there is a constant $K(\gamma, \delta)$ such that, for $n \in \mathbf{N}$,

$$
\left|P_{n}^{(\gamma, \delta)}(x)\right| \leq K(\gamma, \delta) n^{-1 / 2}\left(1-x+n^{-2}\right)^{-\gamma / 2-1 / 4}, \quad x \in[0,1]
$$

(see [9, equation (2.2)] and [10, Theorem 7.32.2]). We shall use (4.20) and (4.21) to estimate

$$
T_{j, n}^{\gamma, \delta} f(x)=\int_{-1}^{1} H_{j}^{\gamma, \delta}(n ; x, y) \omega_{\gamma, \delta}(x) \omega_{\gamma, \delta}(y) \frac{\omega(x)}{\omega(y)} f(y) d y
$$

for $j=1,2$, and

$$
S_{2, n}^{\gamma, \delta} f(x)=\int_{-1}^{1} H_{2}^{\gamma, \delta}(n ; y, x) \omega_{\gamma, \delta}(x) \omega_{\gamma, \delta}(y) \frac{\omega(x)}{\omega(y)} f(y) d y .
$$

We shall begin by considering the operator $T_{2, n}^{\gamma, \delta}$ in some detail.

Note first that, for $n \in \mathbf{N}$ and $x, y \in[-1,1]$, we have

$$
H_{2}^{\gamma, \delta}(n ; x, y) \omega_{\gamma, \delta}(x) \omega_{\gamma, \delta}(y)=b_{1}^{\gamma, \delta}(n ; x) b_{2}^{\gamma, \delta}(n ; y) \frac{w(x)}{w(y)} \frac{1}{x-y}
$$

where

$$
\begin{gathered}
w(x)=\left(1-x^{2}\right)^{-1 / 4}, \\
b_{1}^{\gamma, \delta}(n ; x)=(1-x)^{\gamma / 2+1 / 4}(1+x)^{\delta / 2+1 / 4} n^{1 / 2} P_{n}^{(\gamma, \delta)}(x), \\
b_{2}^{\gamma, \delta}(n ; y)=(1-y)^{\gamma / 2+3 / 4}(1+y)^{\delta / 2+3 / 4} n^{1 / 2} P_{n-1}^{(\gamma+1, \delta+1)}(y) .
\end{gathered}
$$


We shall restrict our attention to $n \geq 2$ (for $n=0,1$ we need only consider operators of the form $T_{1, n}^{\gamma, \delta} ; T_{2, n}^{\gamma, \delta}$ and $S_{2, n}^{\gamma, \delta}$ need not be considered).

For $x \in[-1,0],(4.20)$ and (4.21) imply that

(4.28) $\left|b_{1}^{\gamma, \delta}(n ; x)\right| \leq(1-x)^{\gamma / 2+1 / 4}(1+x)^{\delta / 2+1 / 4} K(\delta, \gamma)\left(1+x+n^{-2}\right)^{-\delta / 2-1 / 4}$;

since $\delta \geq-\frac{1}{2}$, we have $\delta / 2+1 / 4 \geq 0$ so that

$$
\left|b_{1}^{\gamma, \delta}(n ; x)\right| \leq(1-x)^{\gamma / 2+1 / 4} K(\gamma, \delta) \leq 2^{\gamma / 2+1 / 4} K(\delta, \gamma) .
$$

For $x \in[0,1],(4.21)$ implies that

$$
\left|b_{1}^{\gamma, \delta}(n ; x)\right| \leq(1-x)^{\gamma / 2+1 / 4}(1+x)^{\delta / 2+1 / 4} K(\gamma, \delta)\left(1-x+n^{-2}\right)^{-\gamma / 2-1 / 4}
$$

since $\gamma \geq-\frac{1}{2}$, we have $\gamma / 2+1 / 4 \geq 0$ so that

$$
\left|b_{1}^{\gamma, \delta}(n ; x)\right| \leq(1+x)^{\delta / 2+1 / 4} K(\gamma, \delta) \leq 2^{\delta / 2+1 / 4} K(\gamma, \delta) .
$$

Similarly, for $y \in[-1,0],(4.20)$ and (4.21) imply that

$$
\begin{aligned}
&\left|b_{2}^{\gamma, \delta}(n ; y)\right| \leq(1-y)^{\gamma / 2+3 / 4}(1+y)^{\delta / 2+3 / 4} n^{1 / 2}(n-1)^{-1 / 2} \\
& \times K(\delta+1, \gamma+1)\left[1+y+(n-1)^{-2}\right]^{-\delta / 2-3 / 4} \\
& \leq 2^{1 / 2} K(\delta+1, \gamma+1)(1-y)^{\gamma / 2+3 / 4} \\
& \times(1+y)^{\delta / 2+3 / 4}\left[1+y+(n-1)^{2}\right]^{-\delta / 2-3 / 4}
\end{aligned}
$$

since $\delta>-1$, we have $\delta / 2+3 / 4>1 / 4 \geq 0$, so that

(4.33) $\left|b_{2}^{\gamma, \delta}(n ; y)\right| \leq 2^{1 / 2} K(\delta+1, \gamma+1)(1-y)^{\gamma / 2+3 / 4} \leq 2^{\gamma / 2+5 / 4} K(\delta+1, \gamma+1)$.

For $y \in[0,1],(4.21)$ implies that

$$
\begin{aligned}
&\left|b_{2}^{\gamma, \delta}(n ; y)\right| \leq(1-y)^{\gamma / 2+3 / 4}(1+y)^{\delta / 2+3 / 4} n^{1 / 2}(n-1)^{-1 / 2} \\
& \times K(\gamma+1, \delta+1)\left[1-y+(n-1)^{-2}\right]^{-\gamma / 2-3 / 4} \\
& \leq 2^{1 / 2} K(\gamma+1, \delta+1)(1+y)^{\delta / 2+3 / 4}(1-y)^{\gamma / 2+3 / 4} \\
& \times\left[1-y+(n-1)^{-2}\right]^{-\gamma / 2-3 / 4}
\end{aligned}
$$

since $\gamma>-1$, we have $\gamma / 2+3 / 4>1 / 4 \geq 0$, so that

(4.35) $\left|b_{2}^{\gamma, \delta}(n ; y)\right| \leq 2^{1 / 2} K(\gamma+1, \delta+1)(1+y)^{\delta / 2+3 / 4} \leq 2^{\delta / 2+5 / 4} K(\gamma+1, \delta+1)$.

Consequently, the functions $b_{j}^{\gamma, \delta}(n ; \cdot), j=1,2$, are uniformly bounded for $n \geq 2$. Letting $M_{j, n}^{\gamma, \delta}$ denote the operator of multiplication by $b_{j}^{\gamma, \delta}(n ; \cdot)$, we have

$$
T_{2, n}^{\gamma, \delta} f=M_{1, n}^{\gamma, \delta}\left(M_{\omega w} H M_{\omega w}^{-1}\right) M_{2, n}^{\gamma, \delta} \chi_{[-1,1]} f
$$

where $H$ denotes the Hilbert transform. Now it is easily seen that $w^{2}$ is an $A_{2}$ weight; by Lemma 4.1 there exist constants $\delta_{1}, C>0$ depending only upon 
$w$ such that if $\beta \in B M O$ with $\|\beta\|_{*}<\delta_{1}$ then $(\omega w)^{2}$ is also in $A_{2}$ with an $A_{2}$ constant depending only upon $w$. Consequently, for $\|\beta\|_{*}<\delta_{1}$,

$$
\left\|M_{\omega w} H M_{\omega w}^{-1}\right\|_{\mathscr{L}(0)} \leq C
$$

where $C$ is a constant depending only upon $w$ (see [7]). From (4.36) and (4.37) it follows that $\left\|T_{2, n}^{\gamma, \delta}\right\|_{\mathscr{L}(0)}$ is bounded by a constant independent of $n$ and $\beta$.

The analysis of $S_{2, n}^{\gamma, \delta}$ (for $n \geq 2$ ) is similar. It can be seen without difficulty that

$$
H_{2}^{\gamma, \delta}(n ; y, x) \omega_{\gamma, \delta}(x) \omega_{\gamma, \delta}(y)=b_{2}^{\gamma, \delta}(n ; x) b_{1}^{\gamma, \delta}(n ; y) \frac{w^{-1}(x)}{w^{-1}(y)} \frac{1}{y-x}
$$

so that

$$
S_{2, n}^{\gamma, \delta} f=-M_{2, n}^{\gamma, \delta}\left(M_{\omega w^{-1}} H M_{\omega w^{-1}}^{-1}\right) M_{1, n}^{\gamma, \delta} \chi_{[-1,1]} f
$$

since $w^{-2}$ is an $A_{2}$ weight, applying Lemma 4.1 as before shows that for $\|\beta\|_{*}<\delta_{1},\left\|S_{2, n}^{\gamma, \delta}\right\|_{\mathscr{L}(0)}$ is bounded by a constant independent of $n, \beta$.

The analysis of $T_{1, n}^{\gamma, \delta}$ is somewhat easier. Since $\gamma, \delta \geq-\frac{1}{2}$, it is easy to see that for $x, y \in[-1,1], n \in \mathbf{N}$,

$$
\left|H_{1}^{\gamma, \delta}(n ; x, y) \omega_{\gamma, \delta}(x) \omega_{\gamma, \delta}(y)\right| \leq C(\gamma, \delta) w(x) w(y)
$$

where $C(\gamma, \delta)$ is independent of $n$. Then, by (4.22) and (4.40),

$$
\int_{-1}^{1}\left|T_{1, n}^{\gamma, \delta} f(x)\right|^{2} d x \leq C(\gamma, \delta)^{2} \int_{-1}^{1}\left|\int_{-1}^{1} w(x) w(y) \omega(x) \omega(y)^{-1} f(y) d y\right|^{2} d x
$$

Letting $I=[-1,1], b=\log w$, we may write

$$
\begin{gathered}
w(x) w(y) \omega(x) \omega(y)^{-1}=\exp \left[(b+\beta)(x)-m_{I}(b+\beta)\right] \\
\times \exp \left[(b-\beta)(y)-m_{I}(b-\beta)\right] \exp \left[m_{I}(2 b)\right] .
\end{gathered}
$$

Then, by (4.42) and Schwarz' inequality

$$
\begin{aligned}
& \int_{I}\left|\int_{I} w(x) w(y) \omega(x) \omega(y)^{-1} f(y) d y\right|^{2} d x \\
& \leq \exp \left[m_{I}(4 b)\right] \cdot\left(\int_{I} \exp \left[2(b+\beta)(x)-m_{I}(2(b+\beta))\right] d x\right) \\
& \quad \times\left(\int_{I} \exp \left[2(b-\beta)(y)-m_{I}(2(b-\beta))\right] d y\right)\|f\|_{L(0)}^{2} .
\end{aligned}
$$

When $\|\beta\|_{*}<\delta_{1}$, we have $w^{2} \omega^{2}$ and $w^{2} \omega^{-2} \in A_{2}$, so the right-hand side of (4.43) is bounded by a constant times $\|f\|_{L(0)}^{2}$, where the constant depends only on $w$. Thus $\|\beta\|_{*}<\delta_{1}$ implies that $\left\|T_{1, n}^{\gamma, \delta}\right\|_{\mathscr{L}(0)}$ is bounded by a constant independent of $n, \beta$. 
By virtue of the decomposition (4.17), the proof is complete.

The following corollaries are immediate:

Corollary 4.2.1. $\left\langle P_{n}^{\gamma, \delta}\right\rangle$ and $\left\langle Q_{n}^{\gamma, \delta}\right\rangle$ are uniformly holomorphic in a neighborhood of 0 in BMO whenever $\gamma, \delta \geq-\frac{1}{2}$.

Corollary 4.2.2. For $\gamma, \delta \geq-\frac{1}{2}$, there exists a constant $C(\gamma, \delta)$ such that for all $\beta \in \mathbf{B M O}$,

$$
\left\|\left[M_{\beta}, S_{n}^{\gamma, \delta}(0)\right]\right\|_{\mathscr{L}_{;, \delta}(0)} \leq C(\gamma, \delta)\|\beta\|_{*}
$$

\section{AN APPlication to the TODA flow}

Let $d \mu$ be a nonnegative measure on $[-1,1]$ which is absolutely continuous with respect to Lebesgue measure; for example, $d \mu$ may be Lebesgue measure weighted by a Jacobi weight. Following the notation of $\S 2$, for $n \in \mathbf{N}$, let $H_{n}(0)$ denote the set of polynomials of degree at most $n$, considered as a subspace of $L(0)=L^{2}([-1,1], d \mu)$. Let $\omega$ be a fixed nonnegative real-valued function such that $\left.\omega^{2}+\omega^{-2} \in L^{1}([-1,1]), d \mu\right)$; write $\beta=\log \omega \in L^{1}([-1,1], d \mu)$. For each $t$ in a neighborhood of 0 in $\mathbf{R}$, we consider the Gram-Schmidt procedure in the space $L(t \beta)=L^{2}\left([-1,1], \omega^{2 t} d \mu\right)$. Specifically, we let $\left\langle p_{n, t}(x): n \in\right.$ N) denote the orthogonal polynomial system on $L(t \beta)$ obtained by applying the Gram-Schmidt procedure to $\left\langle 1, x, x^{2}, \ldots\right\rangle$. For $t \neq 0$, it is easily seen that $\left\langle p_{n, t}\right\rangle$ arises also by applying Gram-Schmidt to $\left\langle p_{n, 0}\right\rangle$; and, in fact,

$$
p_{n, t}=\frac{\left\{S_{n}(t \beta)-S_{n-1}(t \beta)\right\} p_{n, 0}}{\left\|\left\{S_{n}(t \beta)-S_{n-1}(t \beta)\right\} p_{n, 0}\right\|_{L(t \beta)}} .
$$

The polynomials $\left\langle p_{n, t}\right\rangle$ satisfy the following three-term recurrence (see, for example, $[10, \S 3.2])$ :

$$
x p_{n, t}(x)=A_{n-1}(t) p_{n-1, t}(x)+B_{n}(t) p_{n, t}(x)+A_{n}(t) p_{n+1, t}(x), \quad n \in \mathbf{N}
$$

where we let $p_{-1, t}(x) \equiv 0 \equiv A_{-1}(t)$, and, for $n \in \mathbf{N}$,

$$
\begin{gathered}
A_{n}(t)=\int_{-1}^{1} x\left(M_{\omega^{\prime}} p_{n, t}\right)(x)\left(M_{\omega^{\prime}} p_{n+1, t}\right)(x) d \mu(x), \\
B_{n}(t)=\int_{-1}^{1} x\left[\left(M_{\omega^{\prime}} p_{n, t}\right)(x)\right]^{2} d \mu(x) .
\end{gathered}
$$

The Gram-Schmidt process can be done so that $A_{n}(t)>0$ for all $n \in \mathbf{N}$. It is easy to see that, for $n \in \mathbf{N}$ and $t \in \mathbf{R},\left|A_{n}(t)\right|,\left|B_{n}(t)\right| \leq 1$. We note for future reference that, by (5.1),

$$
M_{\omega^{\prime}} p_{n, t}=\frac{\left\{Q_{n}(t \beta)-Q_{n-1}(t \beta)\right\}\left(M_{\omega^{\prime}} p_{n, 0}\right)}{\left\|\left\{Q_{n}(t \beta)-Q_{n-1}(t \beta)\right\}\left(M_{\omega^{\prime}} p_{n, 0}\right)\right\|_{L(0)}} .
$$


Let $l_{+}^{2}$ denote the complex Hilbert space of square summable sequences; i.e., a sequence $\left\langle a_{n}\right\rangle$ is in $l_{+}^{2}$ if and only if

$$
\left\|\left\langle a_{n}\right\rangle\right\|_{l_{+}^{2}} \equiv \sum_{n=0}^{\infty}\left|a_{n}\right|^{2}<\infty ;
$$

the inner product on $l_{+}^{2}$ is given by

$$
\left(\left\langle a_{n}\right\rangle,\left\langle b_{n}\right\rangle\right)=\sum_{n=0}^{\infty} a_{n} \bar{b}_{n} .
$$

The mapping $L_{t}: L(t \beta) \rightarrow L(t \beta)$ given by $L_{t} f(x)=x f(x)$ induces a bounded linear transformation on $l_{+}^{2}$ given by the matrix

$$
J(t)=\left(\begin{array}{cccccc}
B_{0}(t) & A_{0}(t) & 0 & 0 & \ldots & \ldots \\
A_{0}(t) & B_{1}(t) & A_{1}(t) & 0 & \ldots & \ldots \\
0 & A_{1}(t) & B_{2}(t) & A_{2}(t) & \ldots & \ldots \\
0 & 0 & A_{2}(t) & B_{3}(t) & A_{3}(t) & \ldots \\
& & \ddots & \ddots & \ddots & \\
& & & \ddots & \ddots & \ddots \\
& & & & \ddots & \ddots
\end{array}\right) ;
$$

$J(t)$ is an infinite, symmetric, tridiagonal matrix with strictly positive offdiagonal elements, i.e., a Jacobi matrix. The mapping $t \mapsto J(t)$ defines a flow on the space of Jacobi matrices, which is a generalized infinite-dimensional Toda flow of the type studied by Deift, Li, and Tomei in [4], especially $\S 5$. Deift et. al. have asked for a characterization of those functions $\omega$ for which the flow $J$ is analytic in a neighborhood of 0 in $\mathbf{R}$. As an application of our earlier work, we can now give a partial answer to their question. We remark that the analyticity of the flow $J$ is essentially equivalent to the analyticity of the Gram-Schmidt process relative to measures of the form $\omega^{2 t} d \mu$ on $[-1,1]$.

Let us be more explicit about the operator $J(t)$ on $l_{+}^{2}$. Suppose that $\hat{f}=$ $\left\langle\hat{f}_{0}, \hat{f}_{1}, \hat{f}_{2}, \ldots\right\rangle, \hat{g}=\left\langle\hat{g}_{0}, \hat{g}_{1}, \hat{g}_{2}, \ldots\right\rangle \in l_{+}^{2} ; \hat{f}$ and $\hat{g}$ give the Fourier coefficients for functions $f_{t}, g_{t} \in L(t \beta)$ defined by

$$
f_{t}=\sum_{k=0}^{\infty} \hat{f}_{k} p_{k, t}, \quad g_{t}=\sum_{k=0}^{\infty} \hat{g}_{k} p_{k, t} .
$$

Then we have

$$
\begin{aligned}
(J(t) \hat{f}, \hat{g})= & B_{0}(t) \hat{f}_{0} \overline{\hat{g}}_{0}+A_{0}(t) \hat{f}_{1} \overline{\hat{g}}_{0} \\
& +\sum_{k=0}^{\infty}\left\{A_{k}(t) \hat{f}_{k} \overline{\hat{g}}_{k+1}+B_{k+1} \hat{f}_{k+1} \overline{\hat{g}}_{k+1}+A_{k+1}(t) \hat{f}_{k+1} \overline{\hat{g}}_{k+1}\right\} .
\end{aligned}
$$

We state our question about the analyticity of $J$ precisely, as follows. We would like to know: under what conditions on $\omega$ is it possible to extend $J$ to 
a neighborhood $U$ of 0 in $\mathbf{C}$, in such a way as to insure that the extension $\tilde{J}$ is a holomorphic map from $U$ to the space $\mathscr{L}\left(l_{+}^{2}\right)$ of bounded linear operators on $l_{+}^{2}$ ? By virtue of (5.10), we see that it suffices to obtain conditions on $\omega$ which will insure that there is a neighborhood $U$ of 0 in $\mathbf{C}$ to which, for each $n \in \mathbf{N}$, the functions $A_{n}$ and $B_{n}$ can be holomorphically extended to functions of modulus $\leq 1$.

The extension of $A_{n}$ and $B_{n}$ to complex values is easily effected by defining, for $z \in \mathbf{C}$,

$$
M_{\omega=} p_{n, z}=\frac{\left\{Q_{n}(z \beta)-Q_{n-1}(z \beta)\right\}\left(M_{\omega^{z}} p_{n, 0}\right)}{\left\|\left\{Q_{n}(z \beta)-Q_{n-1}(z \beta)\right\}\left(M_{\omega^{z}} p_{n, 0}\right)\right\|_{L(0)}} ;
$$

the extension is meaningful whenever the extension of $\left\langle Q_{n}\right\rangle$ to complex-valued functions is meaningful. Since the $L(0)$-norm of $(5.11)$ is 1 , it follows from (5.3) and (5.4) with $z$ in place of $t$ that $\left|A_{n}(z)\right|,\left|B_{n}(z)\right| \leq 1$.

In fact, it suffices to obtain conditions on $\omega$ which will guarantee the existence of a neighborhood $U$ of 0 in $\mathrm{C}$ such that the mapping $z \mapsto M_{\omega^{z}} p_{n, z}$ is a holomorphic map from $U$ to $L(0)$ for each $n \in \mathbf{N}$. In the remainder of this section, we will do this in the special case in which $d \mu$ is Lebesgue measure weighted by a Jacobi weight $\omega_{\gamma, \delta}^{2}$, where $\gamma, \delta \geq-\frac{1}{2}$.

We shall make use of the notation established in $\S 4$. For $n \in \mathbf{N}$ and $x \in$ $[-1,1]$, we let

$$
p_{n}^{(\gamma, \delta)}(x)=\frac{P_{n}^{(\gamma, \delta)}(x)}{\left\|P_{n}^{(\gamma, \delta)}\right\|_{L_{\gamma, \delta}}(0)}
$$

denote the $n$th normalized Jacobi polynomial. We begin with the following result:

Lemma 5.1. Suppose $\beta \in B M O$ and let $\omega=e^{\beta}$. For $n \in \mathbf{N}, \gamma, \delta \geq-\frac{1}{2}$, and $z \in \mathbf{C}$, let

$$
F_{n}^{\gamma, \delta}(z)=M_{\omega^{z}} p_{n}^{(\gamma, \delta)}
$$

Then there exists a neighborhood $U$ of 0 in $\mathbf{C}$, and a constant $K>0$, such that for each $n \in \mathbf{N}, F_{n}^{\gamma, \delta}$ is an analytic function from $U$ to $L_{\gamma, \delta}(0)$, and for all $z \in U$,

$$
\left\|F_{n}^{\gamma, \delta}(z)\right\|_{L_{\gamma, \delta}(0)} \leq K .
$$

Proof. By virtue of (4.20), (4.21), and [10, equation (4.3.4), p. 68], we see that there is a constant $K(\gamma, \delta)$ such that, for $n \in \mathbf{N}$,

$$
\left|p_{n}^{(\gamma, \delta)}(x)\right| \leq \begin{cases}K(\gamma, \delta)\left(1+x+n^{-2}\right)^{-\delta / 2-1 / 4}, & x \in[-1,0], \\ \left.K(\gamma, \delta)(1-x+n)^{-2}\right)^{-\gamma / 2-1 / 4}, & x \in[0,1] .\end{cases}
$$


Now note that if $r, s>1$ and $1 / r+1 / s=1$, we have

$$
\begin{aligned}
\left\|F_{n}^{\gamma, \delta}(z)\right\|_{L_{i, \delta}(0)} \leq & \left(\int_{-1}^{1}\left|\omega(x)^{2 z r}\right|(1-x)^{\gamma}(1+x)^{\delta} d x\right)^{1 / r} \\
& \times\left(\int_{-1}^{1}\left|p_{n}^{(\gamma, \delta)}(x)\right|^{2 s}(1-x)^{\gamma}(1+x)^{\delta} d x\right)^{1 / s}
\end{aligned}
$$

by Hölder's inequality. Now we have, by (5.15), for $n \geq 1$,

$$
\begin{aligned}
& \int_{0}^{1}\left|p_{n}^{(\gamma, \delta)}(x)\right|^{2 s}(1-x)^{\gamma}(1+x)^{\delta} d x \\
& \leq 2^{\delta} K(\gamma, \delta)^{2 s} \int_{0}^{1}\left(1-x+n^{-2}\right)^{-\gamma s-s / 2}(1-x)^{\gamma} d x \\
& \leq 2^{\delta+1} K(\gamma, \delta)^{2 s}\left\{\int_{0}^{1-n^{-2}}(1-x)^{-\gamma s-s / 2+\gamma} d x+\int_{1-n^{-2}}^{1} n^{2 \gamma s+s}(1-x)^{\gamma} d x\right\} \\
& \quad=2^{\delta+1} K(\gamma, \delta)^{2 s}\left\{\int_{n^{-2}}^{1} y^{-\gamma s-s / 2+\gamma} d y+n^{2 \gamma s+s} \int_{0}^{n^{-2}} y^{\gamma} d y\right\} .
\end{aligned}
$$

If $\gamma=-\frac{1}{2}$, the rightmost expression in (5.17) becomes

$$
2^{\delta+1} K(\gamma, \delta)^{2 s} \int_{0}^{1} y^{\gamma} d y=(\gamma+1)^{-1} 2^{\delta+1} K(\gamma, \delta)^{2 s} .
$$

If $\gamma>-\frac{1}{2}$ and $1<s<(2+2 \gamma)(1+2 \gamma)^{-1}$, we have $-\gamma s-s / 2+\gamma>-1$, so that the rightmost expression in $(5.17)$ is dominated by

$$
2^{\delta+1} K(\gamma, \delta)^{2 s}\left\{\frac{2}{2 \gamma+2-2 \gamma s-s}+\frac{1}{\gamma+1}\right\} .
$$

Thus it is not difficult to see that for $\gamma, \delta \geq \frac{1}{2}$ and for $s$ satisfying $1<s<$ $\min \left\{(2+2 \gamma)(1+2 \gamma)^{-1},(2+2 \delta)(1+2 \delta)^{-1}\right\}$, there is a constant $K_{1}(\gamma, \delta, s)$ such that for all $n \in \mathbf{N}$,

$$
\left(\int_{-1}^{1}\left|p_{n}^{(\gamma, \delta)}(x)\right|^{2 s}(1-x)^{\gamma}(1+x)^{\delta} d x\right)^{1 / s} \leq K_{1}(\gamma, \delta, s) .
$$

For any such choice of $s$, let $r$ be the conjugate exponent to $s$. By Lemma 4.1, there exists a neighborhood $U_{0}$ of 0 in $\mathbf{C}$ such that, for all $z \in U_{0}$,

$$
\left|\omega(x)^{2 z r}\right|(1-x)^{\gamma}(1+x)^{\delta}
$$

is an $A_{2}$ weight, hence integrable on $[-1,1]$; in fact, using the characterization of $A_{2}$ given in the proof of Lemma 4.1, it follows that for $z \in U_{0}$,

$$
\int_{-1}^{1}\left|\omega(x)^{2 z r}\right|(1-x)^{\gamma}(1+x)^{\delta} d x
$$

is bounded above by a constant independent of $z$. Thus there is a constant $K$ such that for all $z \in U_{0}$, and for all $n \in \mathbf{N}$, we obtain (5.14). 
Now note that

$$
\frac{d}{d z} F_{n}^{\gamma, \delta}(z)=M_{\beta} M_{\omega=} p_{n}^{(\gamma, \delta)}
$$

as before, if $1<s<\min \left\{(2+2 \gamma)(1+2 \gamma)^{-1},(2+2 \delta)(1+2 \delta)^{-1}\right\}$ and $1 / r+1 / s=$ 1 , we obtain

$$
\begin{aligned}
& \left\|\frac{d}{d z} F_{n}^{\gamma, \delta}(z)\right\|_{L_{i, \delta}(0)} \\
& \quad \leq K_{1}(\gamma, \delta, s)\left(\int_{-1}^{1}\left|\beta(x)^{2 z r} \omega(x)^{2 z r}\right|(1-x)^{\gamma}(1+x)^{\delta} d x\right)^{1 / r} \\
& \quad \leq K_{1}(\gamma, \delta, s)\left(\int_{-1}^{1} \exp (4 r|z \beta(x)|) \cdot(1-x)^{\gamma}(1+x)^{\delta} d x\right)^{1 / r} .
\end{aligned}
$$

Again, there is a neighborhood $V_{0}$ of 0 in $\mathbf{C}$ such that, for all $z \in V_{0}$,

$$
\int_{-1}^{1} \exp (4 r|z \beta(x)|) \cdot(1-x)^{\gamma}(1+x)^{\delta} d x
$$

is bounded above by a constant independent of $z$. If we take $U=U_{0} \cap V_{0}$, the lemma follows.

Now suppose $\beta$ is a fixed function in $B M O, \omega=e^{\beta}, \gamma, \delta \geq-\frac{1}{2}$. For $z \in \mathbf{C}$, define

$$
M_{\omega=} p_{n, z}^{(\gamma, \delta)}=\frac{\left\{Q_{n}^{\gamma, \delta}(z \beta)-Q_{n-1}^{\gamma, \delta}(z \beta)\right\}\left(M_{\omega=} p_{n}^{(\gamma, \delta)}\right)}{\left\|\left\{Q_{n}^{\gamma, \delta}(z \beta)-Q_{n-1}^{\gamma, \delta}(z \beta)\right\}\left(M_{\omega=} p_{n}^{(\gamma, \delta)}\right)\right\|_{L_{\gamma, \delta}(0)}} .
$$

By Corollary 4.2.1 and Lemma 5.1, there is a neighborhood $U_{1}$ of 0 in $\mathbf{C}$, and a constant $K_{1}>0$, such that for $n \in \mathbf{N}$, the map

$$
z \mapsto\left\{Q_{n}^{\gamma, \delta}(z \beta)-Q_{n-1}^{\gamma, \delta}(z \beta)\right\}\left(M_{\omega} p_{n}^{(\gamma, \delta)}\right)
$$

is an analytic function from $U_{1}$ to $L_{\gamma, \delta}(0)$, and for all $z \in U_{1}$,

$$
\left\|\left\{Q_{n}^{\gamma, \delta}(z \beta)-Q_{n-1}^{\gamma, \delta}(z \beta)\right\}\left(M_{\omega} p_{n}^{(\gamma, \delta)}\right)\right\|_{L_{\gamma, \delta}(0)} \leq K_{1} .
$$

In particular, this implies that the family of maps (5.27) is continuous on $U_{1}$ uniformly in $n$. Now note that

$$
\left\{Q_{n}^{\gamma, \delta}(0)-Q_{n-1}^{\gamma, \delta}(0)\right\}\left(p_{n}^{(\gamma, \delta)}\right)=p_{n}^{(\gamma, \delta)}
$$

so that, for $z=0$, the denominator in (5.26) is identically 1 . Thus there is a neighborhood $U_{2}$ of 0 in $\mathbf{C}$, and a constant $K_{2}>0$, such that for all $z \in U_{2}$ and for all $n \in \mathbf{N}$,

$$
\left\|\left\{Q_{n}^{\gamma, \delta}(z \beta)-Q_{n-1}^{\gamma, \delta}(z \beta)\right\}\left(M_{\omega=} p_{n}^{(\gamma, \delta)}\right)\right\|_{L_{\gamma, \delta}(0)} \geq K_{2} .
$$

From this, then, it follows that there is a neighborhood $U$ of 0 in $\mathbf{C}$ such that the mapping

$$
z \mapsto M_{\omega} p_{n, z}^{(\gamma, \delta)}
$$

is holomorphic from $U$ to $L_{\gamma, \delta}(0)$ for each $n \in \mathbf{N}$. Consequently, we obtain 
Proposition 5.2. Let $\gamma, \delta \geq-\frac{1}{2}$, let $\beta \in B M O$, and let $\omega=e^{\beta}$. Let $t \mapsto J_{\gamma, \delta}(t)$ denote the Toda flow corresponding to the Gram-Schmidt process relative to the measure

$$
\omega(x)^{2 t}(1-x)^{\gamma}(1+x)^{\delta} d x
$$

on $[-1,1]$. Then there is a neighborhood $U$ of 0 in $\mathbf{C}$ to which $J_{\gamma, \delta}$ may be extended to a holomorphic map $\widetilde{J}_{\gamma, \delta}: U \rightarrow \mathscr{L}\left(l_{+}^{2}\right)$.

\section{UNIFORM ANALYTICITY ON THE CIRCLE, REVISITED}

In $\S 4$, we showed that, for $\gamma, \delta \geq-\frac{1}{2}$, the family $\left\langle P_{n}^{\gamma, \delta}\right\rangle$ of conjugated partial sum operators for Jacobi series is uniformly holomorphic in a neighborhood of 0 in BMO. We conjecture that, in fact, BMO is the space of uniform holomorphy at 0 for $\left\langle P_{n}^{\gamma, \delta}\right\rangle$. Owing in part to the complicated form which the kernel of $P_{n}^{\gamma, \delta}$ takes, this conjecture will be somewhat more difficult to establish than the analogous results for the partial sums of Fourier series which we obtained in $\S 3$.

There are a number of classical results on the equiconvergence of Jacobi series with cosine series (for example, [10, Theorem 9.1.2]), which lead us to consider, as a preliminary step, the problem of determining the space of uniform holomorphy at 0 for the conjugated partial sums of cosine series. In this section, we shall show that $\mathbf{B M O}([0, \pi])$-i.e., the space of even functions of bounded mean oscillation on $\mathrm{T}$-is the space of uniform holomorphy at 0 for conjugated partial sums of cosine series.

We will use the notation established in $\S 3$, with some additions and modifications. Let $\widetilde{L}(0)=L^{2}([0, \pi], d \theta)$ and let $\omega$ be a nonnegative weight function on $[0, \pi]$ such that $\omega, \omega^{-1} \in \widetilde{L}(0)$; write $\beta=\log \omega$. For each $n \in \mathbf{N}$, let $\widetilde{H}_{n}(0)$ be the span of $\langle 1, \cos \theta, \cos 2 \theta, \ldots, \cos n \theta\rangle$ in $\widetilde{L}(0)$. We define $\widetilde{L}(\beta)=L^{2}\left([0, \pi], \omega^{2}(\theta) d \theta\right)$ and let $\widetilde{H}_{n}(\beta)$ denote the closure of $\widetilde{H}_{n}(0)$ in $\widetilde{L}(\beta)$. We let $\widetilde{S}_{n}(\beta)$ be the self-adjoint projection of $\widetilde{L}(\beta)$ onto $\widetilde{H}_{n}(\beta)$, and then define

$$
\begin{gathered}
\widetilde{Q}_{n}(\beta)=M_{\omega} \widetilde{S}_{n}(\beta) M_{\omega}^{-1}, \\
\widetilde{P}_{n}(\beta)=M_{\omega} \widetilde{S}_{n}(0) M_{\omega}^{-1}
\end{gathered}
$$

A function $b \in L^{1}([0, \pi], d \theta)$ is an element of $\mathbf{B M O}([0, \pi], d \theta)$ if and only if

$$
\|b\|_{*} \equiv \sup _{I}|I|^{-1} \int_{I}\left|b(x)-m_{I}(b)\right| d x=\sup _{I} m_{I}\left(\left|b-m_{I}(b)\right|\right)
$$

is finite, where the supremum is taken over all subintervals $I$ of $[0, \pi]$. We shall abbreviate $\mathbf{B M O}([0, \pi], d \theta)$ to $\mathbf{B M O}_{e}$ (the subscript ' $e$ ' stands for 'even'). 
Now suppose that $g \in \widetilde{L}(0)$, and let $\tilde{g}$ be its even extension to $[-\pi, \pi)$. Then

$$
\left.S_{n}(0) \tilde{g}\right|_{[0, \pi]}=\widetilde{S}_{n}(0) g
$$

so that, for $\theta \in[0, \pi]$,

$$
\left\{\widetilde{S}_{n}(0) g\right\}(\theta)=\frac{1}{2 \pi} \int_{-\pi}^{\pi} D_{n}(\theta, \psi) \tilde{g}(\psi) d \psi=\frac{1}{2 \pi} \int_{0}^{\pi} \widetilde{D}_{n}(\theta, \psi) g(\psi) d \psi
$$

where, for $\psi \in[0, \pi]$,

$$
\begin{aligned}
\widetilde{D}_{n}(\theta, \psi) & =D_{n}(\theta,-\psi)+D_{n}(\theta, \psi) \\
& =\frac{\sin \left[(2 n+1) \frac{\theta-\psi}{2}\right]}{\sin \left(\frac{\theta-\psi}{2}\right)}+\frac{\sin \left[(2 n+1) \frac{\theta-\psi}{2}\right]}{\sin \left(\frac{\theta+\psi}{2}\right)} .
\end{aligned}
$$

Letting $\widetilde{\mathscr{L}}(0)$ denote the space of bounded linear operators on $\widetilde{L}(0)$, we obtain the following as an immediate consequence of our work in $\S 3$ :

Proposition 6.1. $\left\langle\widetilde{P}_{n}\right\rangle$ and $\left\langle\widetilde{Q}_{n}\right\rangle$ are uniformly holomorphic families of mappings from a neighborhood of 0 in $\mathbf{B M O}_{e}$ to $\widetilde{\mathscr{L}}(0)$.

To prove that $\mathbf{B M O}_{e}$ is actually the space of uniform holomorphy at 0 for $\left\langle\widetilde{P}_{n}\right\rangle$ and $\left\langle\widetilde{Q}_{n}\right\rangle$, it suffices by Corollary 2.2 .2 to show that $\beta \in \mathbf{B M O}_{e}$ is a necessary and sufficient condition for boundedness of the set $\left\{\left\|\left[M_{\beta}, \widetilde{S}_{n}(0)\right]\right\|_{\tilde{\mathscr{L}}(0)}: n \in\right.$ $\mathbf{N}$ \} . To gain some intuition for this problem, we first consider a somewhat simpler problem involving "partial sums" of Fourier transforms on $\mathbf{R}$.

For $f \in L^{1}(\mathbf{R})$, we define the Fourier transform and its inverse according to the normalization

$$
\tilde{f}(\xi)=\int_{\mathbf{R}} e^{-i x \xi} f(x) d x, \quad \check{f}(x)=\frac{1}{2 \pi} \int_{\mathbf{R}} e^{i x \xi} f(\xi) d \xi .
$$

For each positive integer $n$, we define the operator $T_{n}(0): L^{2}(\mathbf{R}) \rightarrow L^{2}(\mathbf{R})$ by setting

$$
T_{n}(0) f=\left(\hat{f} \chi_{[-n, n]}\right)^{\vee}
$$

for $f \in L^{2}(\mathbf{R}) . T_{n}(0)$ is a convolution operator, with kernel

$$
K_{n}(x)=\frac{1}{\pi x} \sin n x .
$$

We define the operator $\widetilde{T}_{n}(0): L^{2}([0, \infty)) \rightarrow L^{2}([0, \infty))$ as follows: for $g \in$ $L^{2}([0, \infty))$, let $\tilde{g}$ denote its even extension to $\mathbf{R}$, and define

$$
\tilde{T}_{n}(0) g=\left.T_{n}(0) \tilde{g}\right|_{[0, \infty)} .
$$

For $x \in[0, \infty)$, we have

$$
\left\{\widetilde{T}_{n}(0)\right\} g(x)=\int_{\mathbf{R}} K_{n}(x-y) \tilde{g}(y) d y=\int_{0}^{\infty} \widetilde{K}_{n}(x, y) g(y) d y
$$


where

$$
\begin{aligned}
\tilde{K}_{n}(x, y) & =K_{n}(x-y)+K_{n}(x+y) \\
& =\frac{1}{\pi}\left\{\frac{(x+y) \sin n(x-y)+(x-y) \sin n(x+y)}{x^{2}-y^{2}}\right\} .
\end{aligned}
$$

The operators $T_{n}(0)$ and $\widetilde{T}_{n}(0)$ are the continuous analogues of $S_{n}(0)$ and $\tilde{S}_{n}(0)$, respectively. Now suppose that $\omega=e^{\beta}$ is a nonnegative weight function on $\mathbf{R}$ such that $\omega^{2}+\omega^{-2} \in L_{\text {loc }}^{1}(\mathbf{R})$, and define $T_{n}(\beta)=M_{\omega} T_{n}(0) M_{\omega}^{-1}$. We obtain the following continuous analogue of Proposition 3.1:

Proposition 6.2. The quantity $\sup \left\langle\left\|T_{n}(\beta)\right\|_{\mathscr{L}\left(L^{2}(\mathbf{R})\right)}: n=1,2,3, \ldots\right\rangle$ is finite if and only if $\omega^{2} \in A_{2}$.

Proof. This is the content of Corollary 3.1.2, Chapter 4 of [5].

Corollary 6.2.1. $\left\langle T_{n}\right\rangle$ is a uniformly holomorphic family of mappings from a neighborhood of 0 in $\mathbf{B M O}(\mathbf{R})$ to $\mathscr{L}\left(L^{2}(\mathbf{R})\right)$.

Proof. Completely analogous to that of Corollary 3.1.1.

Corollary 6.2.2. $\left\langle\widetilde{T}_{n}\right\rangle$ is a uniformly holomorphic family of mappings from a neighborhood of 0 in $\mathbf{B M O}([0, \infty))$ to $\mathscr{L}\left(L^{2}([0, \infty))\right)$.

It is left as a straightforward exercise for the reader to prove the continuous analogue of Proposition 3.2 (i.e., with $T_{n}(0)$ in place of $\left.S_{n}(0)\right)$. It is the proof of the corresponding result for $\widetilde{T}_{n}(0)$ that is of greatest interest to us here. We shall begin with an extremely useful lemma.

Let $1<p<\infty$. A nonnegative weight function $w$ on $\mathbf{R}$ is said to belong to the class $A_{p}$ if and only if both $w$ and $w^{-1 /(p-1)}$ are locally integrable, and there is a constant $C>0$ such that for all subintervals $I$ of $\mathbf{R}$,

$$
\left(|I|^{-1} \int_{I} w\right)\left(|I|^{-1} \int_{I} w^{-1 /(p-1)}\right)^{p-1} \leq C \text {. }
$$

The smallest constant for which (6.13) holds is called the $A_{p}$ constant of $w$. It is worth noting here that

$$
A_{1}=\bigcap_{1 \leq p<\infty} A_{p}, \quad A_{\infty}=\bigcup_{1 \leq p<\infty} A_{p}
$$

(see, for example, [5, Chapter 4, Theorem 1.14 and Corollary 2.13]). Our lemma is as follows:

Lemma 6.3. Suppose $w \in A_{\infty}$ and $b \in L_{\text {loc }}^{1}(\mathbf{R})$. Suppose, moreover, that there is a constant $K(b)$ such that, for all subintervals $I$ of $\mathbf{R}$,

$$
w(I)^{-1} \int_{I}\left|b(x)-m_{I}(b)\right| w(x) d x \leq K(b)
$$

where

$$
w(I)=\int_{I} w(x) d x
$$


Then $b \in \mathbf{B M O}(\mathbf{R})$, and $\|b\|_{*} \leq C(w) K(b)$, where $C(w)$ is a constant depending only upon $w$.

Proof. Since $w \in A_{\infty}$, it follows from (6.14) that there is a $p \in(1, \infty)$ such that $w \in A_{p}$. Now let $I$ be a subinterval of $\mathbf{R}$; we have, by Hölder's inequality,

$$
\begin{aligned}
& \int_{I}\left|b(x)-m_{I}(b)\right| d x \\
& \quad \leq\left(\int_{I}\left|b(x)-m_{I}(b)\right|^{p} w(x) d x\right)^{1 / p}\left(\int_{I} w(x)^{-1 /(p-1)} d x\right)^{(p-1) / p} .
\end{aligned}
$$

It is not difficult to see that there is a constant $C_{1}$ depending only upon $p$ such that

$$
\left(\int_{I}\left|b(x)-m_{I}(b)\right|^{p} w(x) d x\right)^{1 / p} \leq C_{1} w(I)^{1 / p} K(b)
$$

(see, for example, [5, Chapter 2, Corollary 3.10]). Moreover, if $C_{2}$ is the $A_{p}$ constant of $w$, we have

$$
\left(\int_{I} w(x)^{-1 /(p-1)} d x\right)^{(p-1) / p} \leq C_{2}^{1 / p}|I| w(I)^{-1 / p} .
$$

Combining (6.17)-(6.19), and letting $C(w)=C_{1} C_{2}^{1 / p}$, we have

$$
\int_{I}\left|b(x)-m_{I}(b)\right| d x \leq|I| C(w) K(b)
$$

from which the result follows.

We make use of the lemma to prove

Proposition 6.4. There exist constants $C_{1}, C_{2}>0$ such that

$$
C_{1}\|\beta\|_{*} \leq \sup _{n}\left\|\left[M_{\beta}, \widetilde{T}_{n}(0)\right]\right\|_{\mathrm{op}} \leq C_{2}\|\beta\|_{*}
$$

for all $\beta \in L_{\text {loc }}^{1}([0, \infty))$, where $\|\cdot\|_{*}$ denotes the norm on $\mathbf{B M O}([0, \infty))$, and $\|\cdot\|_{\mathrm{op}}$ denotes the norm as an operator on $L^{2}([0, \infty))$.

Proof. The existence of $C_{2}$ with the requisite property is immediate from Corollary 6.2.2. To establish the existence of $C_{1}$, we make use of Lemma 6.3. For $x \in[0, \infty)$, let $w(x)=x^{2} ; w$ is an $A_{\infty}$ weight. We shall show that there exists a constant $\mu>0$ such that for all subintervals $I$ of $[0, \infty)$, and for all $\beta \in L_{\text {loc }}^{1}([0, \infty))$,

$$
w(I)^{-1} \int_{I}\left|\beta(x)-m_{I}(\beta)\right|^{2} w(x) d x \leq \mu C^{2},
$$

where

$$
C=\sup _{n}\left\|\left[M_{\beta}, \widetilde{T}_{n}(0)\right]\right\|_{\mathrm{op}} .
$$

The result is then immediate from the $[0, \infty)$-version of Lemma 6.3. 
Let $I$ be a subinterval of $[0, \infty)$ and let $x \in[0, \infty)$. Define the function $f_{x}=f_{I, x, n}$ by setting,

$$
f_{x}(y)=\chi_{I}(y) \pi\left(x^{2}-y^{2}\right) \cos n y=\chi_{I}(y) \frac{\pi}{2}\left(x^{2}-y^{2}\right)\left(e^{i n y}+e^{-i n y}\right)
$$

for $y \in[0, \infty)$. Note that, for $y \in I$,

$$
\begin{aligned}
\frac{1}{\pi} \frac{(x+y) \sin n(x-y)}{x^{2}-y^{2}} f_{x}(y) & =\frac{1}{4 i}(x+y)\left[e^{i n(x-y)}-e^{-i n(x-y)}\right]\left(e^{i n y}+e^{-i n y}\right) \\
& =\frac{1}{4 i}(x+y)\left[e^{i n x}-e^{-i n x}-e^{i n(2 y-x)}+e^{-i n(2 y-x)}\right. \\
& =\frac{1}{2}(x+y) \sin n x+\frac{1}{2}(x+y) \sin n(x-2 y),
\end{aligned}
$$

$$
\begin{aligned}
\frac{1}{\pi} \frac{(x-y) \sin n(x+y)}{x^{2}-y^{2}} f_{x}(y) & =\frac{1}{4 i}(x-y)\left[e^{i n(x+y)}-e^{-i n(x+y)}\right]\left(e^{i n y}+e^{-i n y}\right) \\
& =\frac{1}{4 i}(x-y)\left[e^{i n x}-e^{-i n x}-e^{-i n(2 y+x)}+e^{i n(2 y+x)}\right] \\
& =\frac{1}{2}(x-y) \sin n x+\frac{1}{2}(x-y) \sin n(x+2 y),
\end{aligned}
$$

so that, by $(6.12)$,

$$
\begin{aligned}
K_{n}(x, y) f_{x}(y)= & \left\{x \sin n x+\frac{1}{2}(x+y) \sin n(x-2 y)\right. \\
& \left.+\frac{1}{2}(x-y) \sin n(x+2 y)\right\} \chi_{I}(y) \\
= & \{x \sin n x+x \sin n x \cos 2 n y-y \sin 2 n y \cos n x\} \chi_{I}(y) .
\end{aligned}
$$

Combining (6.11) and (6.27), we obtain

$$
\begin{aligned}
& \left\{\left[M_{\beta}, \widetilde{T}_{n}(0)\right] f_{x}\right\}(x)=x \sin n x|I|\left(\beta(x)-m_{I}(\beta)\right) \\
& \quad+x \sin n x \int_{0}^{\infty} \cos 2 n y(\beta(x)-\beta(y)) \chi_{I}(y) d y \\
& \quad-\cos n x \int_{0}^{\infty} y \sin 2 n y(\beta(x)-\beta(y)) \chi_{I}(y) d y .
\end{aligned}
$$

As in the proof of Proposition 3.2, we apply the Riemann-Lebesgue lemma and Fatou's lemma to see that

$$
\begin{aligned}
\varlimsup_{n \rightarrow \infty} \int_{I}\left|\left\{\left[M_{\beta}, \widetilde{T}_{n}(0)\right] f_{x}\right\}(x)\right|^{2} d x & =\varlimsup_{n \rightarrow \infty} \int_{I} x^{2} \sin ^{2} n x|I|^{2}\left|\beta(x)-m_{I}(\beta)\right|^{2} d x \\
& =\frac{1}{2}|I|^{2} \int_{I}\left|\beta(x)-m_{I}(\beta)\right|^{2} w(x) d x
\end{aligned}
$$

where we have used the fact that $\sin ^{2} n x=\frac{1}{2}-\frac{1}{2} \cos 2 n x$.

Now suppose $x_{0}$ is the midpoint of $I$, and suppose further that $x_{0}>2|I|$. By (6.24), we may write

$$
f_{x}(y)=\left(x^{2}-x_{0}^{2}\right) \frac{\pi}{2} \chi_{I}(y) \cos n y+\left(x_{0}^{2}-y^{2}\right) \frac{\pi}{2} \chi_{I}(y) \cos n y
$$


so that

$$
\begin{aligned}
\left\{\left[M_{\beta}, \widetilde{T}_{n}(0)\right] f_{x}\right\}(x)= & \left(x^{2}-x_{0}^{2}\right) \frac{\pi}{2}\left\{\left[M_{\beta}, \widetilde{T}_{n}(0)\right] h_{1}\right\}(x) \\
& +\frac{\pi}{2}\left\{\left[M_{\beta}, \widetilde{T}_{n}(0)\right] h_{2}\right\}(x),
\end{aligned}
$$

where

$$
h_{1}(y)=\chi_{I}(y) \cos n y, \quad h_{2}(y)=\left(x_{0}^{2}-y^{2}\right) \chi_{I}(y) \cos n y .
$$

If $x_{0}>2|I|$, then, for $y \in I$,

$$
\frac{3 x_{0}}{4} \leq x_{0}-\frac{|I|}{2} \leq y \leq x_{0}+\frac{|I|}{2} \leq \frac{5 x_{0}}{4},
$$

so that, in particular,

$$
\left(y^{2}-x_{0}^{2}\right)^{2}=\left(y-x_{0}\right)^{2}\left(y+x_{0}\right)^{2} \leq\left(|I|^{2} / 4\right)\left(81 x_{0}^{2} / 16\right) \leq 2 x_{0}^{2}|I|^{2} .
$$

Consequently,

$$
\begin{gathered}
\left\|h_{1}\right\|_{2}^{2} \leq|I|, \\
\left\|h_{2}\right\|_{2}^{2} \leq \int_{I} 2 x_{0}^{2}|I|^{2} d x=2 x_{0}^{2}|I|^{3}, \\
w(I)=\int_{I} y^{2} d y \geq \frac{9}{16} x_{0}^{2}|I| .
\end{gathered}
$$

Thus, by (6.23), (6.31), and (6.34)-(6.37), we have

$$
\int_{I}\left|\left\{\left[M_{\beta}, \tilde{T}_{n}(0)\right] f_{x}\right\}(x)\right|^{2} d x \leq \pi^{2} x_{0}^{2}|I|^{3} C^{2} \leq \frac{16}{9} \pi^{2} C^{2} w(I)|I|^{2} .
$$

Thus, by (6.29)

$$
w(I)^{-1} \int_{I}\left|\beta(x)-m_{I}(\beta)\right|^{2} w(x) d x \leq \frac{32}{9} \pi^{2} C^{2} .
$$

If, on the other hand, $\frac{1}{2}|I| \leq x_{0} \leq 2|I|$, we write

$$
f_{x}(y)=x^{2} \frac{\pi}{2} \chi_{I}(y) \cos n y-y^{2} \frac{\pi}{2} \chi_{I}(y) \cos n y
$$

so that

$$
\left\{\left[M_{\beta}, \tilde{T}_{n}(0)\right] f_{x}\right\}(x)=x^{2} \frac{\pi}{2}\left\{\left[M_{\beta}, \widetilde{T}_{n}(0)\right] h_{1}\right\}(x)-\frac{\pi}{2}\left\{\left[M_{\beta}, \widetilde{T}_{n}(0)\right] w h_{1}\right\}(x) .
$$

Now we have

$$
\begin{aligned}
w(I)=\int_{I} y^{2} d y & =\frac{1}{3}\left\{\left(x_{0}+\frac{1}{2}|I|\right)^{3}-\left(x_{0}-\frac{1}{2}|I|\right)^{3}\right\} \\
& =\frac{1}{3}\left\{3 x_{0}^{2}|I|+\frac{1}{4}|I|^{3}\right\} \\
& \geq \frac{1}{12}|I|^{3}
\end{aligned}
$$




$$
\begin{gathered}
\left\|h_{1}\right\|_{2}^{2} \leq|I|, \\
\left\|w h_{1}\right\|_{2}^{2} \leq \int_{I} y^{4} d y \leq \frac{1}{5}|I|^{5} .
\end{gathered}
$$

Noting that, for $x \in I, x \leq \frac{5}{2}|I|$, we have

$$
\int_{I}\left|\left\{\left[M_{\beta}, \widetilde{T}_{n}(0)\right] f_{x}\right\}(x)\right|^{2} d x \leq 10 \pi^{2} C^{2}|I|^{5} \leq 120 \pi^{2} C^{2} w(I)|I|^{2},
$$

by (6.23) and (6.41)-(6.44). Hence, by (6.29),

$$
w(I)^{-1} \int_{I}\left|\beta(x)-m_{I}(\beta)\right|^{2} w(x) d x \leq 240 \pi^{2} C^{2} .
$$

Thus, combining (6.39) and (6.46), we obtain (6.22) with $\mu=240 \pi^{2}$. This completes the proof.

Corollary 6.4.1. $\mathrm{BMO}([0, \infty))$ is the space of uniform holomorphy at 0 for $\left\langle\widetilde{T}_{n}\right\rangle$.

The proof of the periodic analogue of Proposition 6.4 is only slightly more complicated, but the basic idea is the same:

Proposition 6.5. Suppose $\beta \in L^{2}([0, \pi])$. Then

$$
C=\sup _{n}\left\|\left[M_{\beta}, \widetilde{S}_{n}(0)\right]\right\|_{\tilde{\mathscr{L}}(0)}<\infty
$$

if and only if $\beta \in \mathbf{B M O}_{e}$.

Proof. The sufficiency of $\beta \in \mathbf{B M O}_{e}$ is an immediate consequence of Proposition 6.1. We shall prove that (6.47) implies $\beta \in \mathbf{B M O}_{e}$ by using the $[0, \pi]-$ version of Lemma 6.3.

For $\theta \in[0, \pi]$, let $w(\theta)=\sin ^{2} \theta / 2 ; w$ is an $A_{\infty}$ weight on $[0, \pi]$. We shall show that there exists a constant $\mu>0$ such that whenever $I$ is a subinterval of $[0, \pi]$ with $|I|<\pi / 400$, and whenever $\beta \in L^{1}([0, \pi])$, there is a constant $c_{I}(\beta)$ such that

$$
w(I)^{-1} \int_{I}\left|\beta(\theta)-c_{I}(\beta)\right|^{2} w(\theta) d \theta \leq \mu C^{2} .
$$

The result then follows from the $[0, \pi]$-version of Lemma 6.3 together with $[5$, Chapter 1, Lemmas 9.4 and 9.5].

Let $I$ be a subinterval of $[0, \pi]$ with $|I|<\pi / 400$, and let $\theta \in I$. Define the function $f_{\theta}=f_{I, \theta, n}$ by setting, for $\psi \in[0, \pi]$,

$$
\begin{aligned}
f_{\theta}(\psi)= & 2 \chi_{I}(\psi) \cos \left[(2 n+1) \frac{\psi}{2}\right] \sin \left(\frac{\theta-\psi}{2}\right) \sin \left(\frac{\theta+\psi}{2}\right) \\
= & \chi_{I}(\psi)\left\{\exp \left[i(2 n+1) \frac{\psi}{2}\right]+\exp \left[-i(2 n+1) \frac{\psi}{2}\right]\right\} \\
& \times \sin \left(\frac{\theta-\psi}{2}\right) \sin \left(\frac{\theta+\psi}{2}\right) .
\end{aligned}
$$


A straightforward calculation using (6.6) shows that

$$
\widetilde{D}_{n}(\theta, \psi) f_{\theta}(\psi)=2 \sin \left[(2 n+1) \frac{\theta}{2}\right] \sin \frac{\theta}{2} \cos \frac{\psi}{2}+\Phi(n, \theta, \psi)
$$

where

$$
\begin{aligned}
\Phi(n, \theta, \psi)= & \sin \left[(2 n+1)\left(\frac{\theta-2 \psi}{2}\right)\right] \sin \left(\frac{\theta+\psi}{2}\right) \\
& +\sin \left[(2 n+1)\left(\frac{\theta+2 \psi}{2}\right)\right] \sin \left(\frac{\theta-\psi}{2}\right) .
\end{aligned}
$$

For $\psi \in[0, \pi]$, let $\rho(\psi)=\cos \psi / 2$, and set

$$
\rho(I)=\int_{I} \rho(\psi) d \psi
$$

Then, by (6.5) and (6.50),

$$
\begin{aligned}
\left\{\left[M_{\beta}, \widetilde{S}_{n}(0)\right] f_{\theta}\right\}(\theta)= & \frac{1}{2 \pi} \int_{0}^{\pi} \widetilde{D}_{n}(\theta, \psi) f_{\theta}(\psi)(\beta(\theta)-\beta(\psi)) d \psi \\
= & \frac{1}{\pi} \sin \left[(2 n+1) \frac{\theta}{2}\right] \sin \frac{\theta}{2} \rho(I)\left[\beta(\theta)-c_{I}(\beta)\right] \\
& +\frac{1}{2 \pi} \int_{I} \Phi(n, \theta, \psi)(\beta(\theta)-\beta(\psi)) d \psi
\end{aligned}
$$

where

$$
c_{I}(\beta)=\rho(I)^{-1} \int_{I} \beta(\psi) \rho(\psi) d \psi
$$

and, by the Riemann-Lebesgue lemma,

$$
\lim _{n \rightarrow \infty} \frac{1}{2 \pi} \int_{I} \Phi(n, \theta, \psi)(\beta(\theta)-\beta(\psi)) d \psi=0 .
$$

Applying the Riemann-Lebesgue lemma and Fatou's lemma in by now familiar fashion, we obtain

$$
\begin{aligned}
\varlimsup_{n \rightarrow \infty} & \left.\int_{I} \mid\left\{M_{\beta}, \widetilde{S}_{n}(0)\right] f_{\theta}\right\}\left.(\theta)\right|^{2} d \theta \\
& =\varlimsup_{n \rightarrow \infty} \frac{1}{\pi^{2}} \rho(I)^{2} \int_{I} \sin ^{2}\left[(2 n+1) \frac{\theta}{2}\right]\left|\beta(\theta)-c_{I}(\beta)\right|^{2} w(\theta) d \theta \\
& =\frac{1}{2 \pi^{2}} \rho(I)^{2} \int_{I}\left|\beta(\theta)-c_{I}(\beta)\right|^{2} w(\theta) d \theta
\end{aligned}
$$

where we have used the fact that $\sin ^{2}\left[(2 n+1) \frac{\theta}{2}\right]=\frac{1}{2}-\frac{1}{2} \cos [(2 n+1) \theta]$.

Now suppose $\psi_{0}$ is the midpoint of $I$. We shall consider five cases:

(i) $\pi / 200>2|I| \geq \psi_{0}$;

(ii) $\pi / 200>\psi_{0}>2|I|$;

(iii) $\pi / 200 \leq \psi_{0} \leq 199 \pi / 200$;

(iv) $\pi / 200>2|I| \geq \pi-\psi_{0}$,i.e., $199 \pi / 200<\pi-2|I| \leq \psi_{0}$;

(v) $\pi / 200>\pi-\psi_{0}>2|I|$, i.e., $199 \pi / 200<\psi_{0}<\pi-2|I|$. 
We begin with:

Case (i): $\pi / 200>2|I| \geq \psi_{0}$. The addition formula for sines show that

$$
\begin{aligned}
\sin \left(\frac{\theta+\psi}{2}\right) \sin \left(\frac{\theta-\psi}{2}\right) & =\sin ^{2} \frac{\theta}{2} \cos ^{2} \frac{\psi}{2}-\sin ^{2} \frac{\psi}{2} \cos ^{2} \frac{\theta}{2} \\
& =w(\theta) \rho^{2}(\psi)-w(\psi) \rho^{2}(\theta) .
\end{aligned}
$$

If we let

$$
\lambda_{n}(\psi)=2 \cos \left[(2 n+1) \frac{\psi}{2}\right] \chi_{I}(\psi)
$$

then, by (6.49),

$$
f_{\theta}(\psi)=w(\theta) \rho^{2}(\psi) \lambda_{n}(\psi)-\rho^{2}(\theta) w(\psi) \lambda_{n}(\psi)
$$

so that

$\left\{\left[M_{\beta}, \widetilde{S}_{n}(0)\right] f_{\theta}\right\}(\theta)=w(\theta)\left\{\left[M_{\beta}, \widetilde{S}_{n}(0)\right] \rho^{2} \lambda_{n}\right\}(\theta)-\rho^{2}(\theta)\left\{\left[M_{\beta}, \widetilde{S}_{n}(0)\right] w \lambda_{n}\right\}(\theta)$.

It is easily seen that there exist constants $C_{1}, C_{2}, C_{3}>0$ such that, for $\psi \in I$,

$$
C_{I}|\psi|^{2} \leq w(\psi) \leq C_{2}|\psi|^{2} \quad \text { and } \quad C_{3} \leq \rho(\psi) \leq 1
$$

Thus we have

$$
\begin{gathered}
|I| \geq \rho(I) \geq C_{3}|I|, \\
\left\|\rho^{2} \lambda_{n}\right\|_{2}^{2} \leq \int_{I} \rho(\psi)^{4} d \psi \leq|I|, \\
\left\|w \lambda_{n}\right\|_{2}^{2} \leq C_{2}^{2} \int_{I} \psi^{4} d \psi=C_{2}^{2} \frac{1}{5}\left(\frac{5}{2}|I|\right)^{5} \leq 20 C_{2}^{2}|I|^{5}, \\
w(I) \geq C_{1} \int_{I} \psi^{2} d \psi \geq \frac{1}{12} C_{1}|I|^{3},
\end{gathered}
$$

where the estimation (6.65) is exactly like (6.42). Note also that, for $\psi \in I$,

$$
w(\psi) \leq C_{2}|\psi|^{2} \leq \frac{25}{4} C_{2}|I|^{2} .
$$

Then we have

$$
\begin{aligned}
\int_{I}\{[ & \left.\left.M_{\beta}, \tilde{S}_{n}(0)\right] f_{\theta}\right\}\left.(\theta)\right|^{2} d \theta \\
& \leq \int_{I}|w(\theta)|^{2}\left|\left\{\left[M_{\beta}, \widetilde{S}_{n}(0)\right] \rho^{2} \lambda_{n}\right\}(\theta)\right|^{2} d \theta+\int_{I}\left|\left\{\left[M_{\beta}, \widetilde{S}_{n}(0)\right] w \lambda_{n}\right\}(\theta)\right|^{2} d \theta \\
& \leq\left[\frac{25}{4} C_{2}|I|^{2}\right]^{2} \cdot C^{2} \cdot\left\|\rho^{2} \lambda_{n}\right\|_{2}^{2}+C^{2} \cdot\left\|w \lambda_{n}\right\|_{2}^{2} \\
& \leq K_{1} w(I) \rho(I)^{2} C^{2}
\end{aligned}
$$

where $K_{1}$ is independent of $\beta, I$, and $n$. Combining (6.56) and (6.67), we obtain

$$
w(I)^{-1} \int_{I}\left|\beta(\theta)-c_{I}(\beta)\right|^{2} w(\theta) d \theta \leq 2 \pi^{2} K_{1} C^{2}
$$


Case (ii): $\pi / 200>\psi_{0}>2|I|$. In this case we write (6.69)

$$
\begin{aligned}
\sin \left(\frac{\theta-\psi}{2}\right) & =\sin \left(\frac{\theta-\psi_{0}+\psi_{0}-\psi}{2}\right) \\
& =\sin \left(\frac{\theta-\psi_{0}}{2}\right) \cos \left(\frac{\psi_{0}-\psi}{2}\right)+\sin \left(\frac{\psi_{0}-\psi}{2}\right) \cos \left(\frac{\theta-\psi_{0}}{2}\right),
\end{aligned}
$$

(6.70) $\sin \left(\frac{\theta+\psi}{2}\right)=\sin \frac{\theta}{2} \cos \frac{\psi}{2}+\cos \frac{\theta}{2} \sin \frac{\psi}{2}$

$$
=w^{1 / 2}(\theta) \rho(\psi)+\rho(\theta) w^{1 / 2}(\psi) .
$$

If we let $w_{0}^{1 / 2}(\theta)=\sin \left(\frac{\theta-\psi_{0}}{2}\right), \rho_{0}(\theta)=\cos \left(\frac{\theta-\psi_{0}}{2}\right)$, then we have

$$
\begin{aligned}
(6.71) \sin & \left(\frac{\theta+\psi}{2}\right) \sin \left(\frac{\theta-\psi}{2}\right) \\
= & {\left[w^{1 / 2}(\theta) \rho(\psi)+\rho(\theta) w^{1 / 2}(\psi)\right]\left[w_{0}^{1 / 2}(\theta) \rho_{0}(\psi)-w_{0}^{1 / 2}(\psi) \rho_{0}(\theta)\right] } \\
= & \left(w w_{0}\right)^{1 / 2}(\theta)\left(\rho \rho_{0}\right)(\psi)-\left(w^{1 / 2} \rho_{0}\right)(\theta)\left(\rho w_{0}^{1 / 2}\right)(\psi) \\
& +\left(w_{0}^{1 / 2} \rho\right)(\theta)\left(\rho_{0} w^{1 / 2}\right)(\psi)-\left(\rho \rho_{0}\right)(\theta)\left(w w_{0}\right)^{1 / 2}(\psi)
\end{aligned}
$$

so that, by (6.49), (6.58), and (6.71), we have

$$
\begin{aligned}
& \left\{\left[M_{\beta}, \tilde{S}_{n}(0)\right] f_{\theta}\right\}(\theta)=\left(w w_{0}\right)^{1 / 2}(\theta)\left\{\left[M_{\beta}, \widetilde{S}_{n}(0)\right] \lambda_{n} \rho \rho_{0}\right\}(\theta) \\
& \quad-\left(w^{1 / 2} \rho_{0}\right)(\theta)\left\{\left[M_{\beta}, \tilde{S}_{n}(0)\right] \lambda_{n} \rho w_{0}^{1 / 2}\right\}(\theta) \\
& \quad+\left(w_{0}^{1 / 2} \rho\right)(\theta)\left\{\left[M_{\beta}, \widetilde{S}_{n}(0)\right] \lambda_{n} \rho_{0} w^{1 / 2}\right\}(\theta) \\
& \quad-\left(\rho \rho_{0}\right)(\theta)\left\{\left[M_{\beta}, \widetilde{S}_{n}(0)\right] \lambda_{n} w^{1 / 2} w_{0}^{1 / 2}\right\}(\theta) .
\end{aligned}
$$

Since $\psi_{0}$ and $|I|$ are small, (6.61) continues to hold for $\psi \in I$, and hence (6.62) holds as well. Moreover, (6.61) holds for $w_{0}, \rho_{0}$ in place of $w, \rho$. As in (6.33), we obtain

$$
\frac{3 \psi_{0}}{4} \leq \psi \leq \frac{5 \psi_{0}}{4}, \quad \psi \in I
$$

Thus we have:

$$
w(I) \geq \frac{9}{16} C_{1} \psi_{0}^{2}|I|
$$

For $\psi \in I$, we have

$$
\begin{gathered}
\left|\left(w w_{0}\right)^{1 / 2}(\psi)\right| \leq \frac{5}{8} C_{2} \psi_{0}|I|, \\
\left|\left(w^{1 / 2} \rho_{0}\right)(\psi)\right| \leq \frac{5}{4} C_{2}^{1 / 2} \psi_{0}, \\
\left|\left(w_{0}^{1 / 2} \rho\right)(\psi)\right| \leq \frac{1}{2} C_{2}^{1 / 2}|I|, \\
\left|\left(\rho \rho_{0}\right)(\psi)\right| \leq 1,
\end{gathered}
$$


and so we have

$$
\begin{gathered}
\left\|\lambda_{n}\left(w w_{0}\right)^{1 / 2}\right\|_{2}^{2} \leq \frac{25}{64} C_{2}^{2} \psi_{0}^{2}|I|^{3}, \\
\left\|\lambda_{n}\left(w^{1 / 2} \rho_{0}\right)\right\|_{2}^{2} \leq \frac{25}{16} C_{2} \psi_{0}^{2}|I|, \\
\left.\| \lambda_{n}\left(w_{0}^{1 / 2} \rho\right)\right) \|_{2}^{2} \leq \frac{1}{4} C_{2}|I|^{3}, \\
\left.\| \lambda_{n}\left(\rho \rho_{0}\right)\right) \|_{2}^{2} \leq|I| .
\end{gathered}
$$

Thus, by (6.62), (6.72), and (6.74)-(6.82) we have

$$
\int_{I}\left|\left\{\left[M_{\beta}, \tilde{S}_{n}(0)\right] f_{\theta}\right\}(\theta)\right|^{2} d \theta \leq K C^{2}|I|^{3} \psi_{0}^{2} \leq K_{2} C^{2} w(I) \rho(I)^{2}
$$

where $K, K_{2}$ are independent of $\beta, I, n$. Combining (6.56) and (6.83), we obtain

$$
w(I)^{-1} \int_{I}\left|\beta(\theta)-c_{I}(\beta)\right|^{2} w(\theta) d \theta \leq 2 \pi^{2} K_{2} C^{2} .
$$

Case (iii): $\pi / 200 \leq \psi_{0} \leq 199 \pi / 200$. Exactly as in Case (ii), we obtain the expression (6.72). For this case, we observe that the functions $w, \rho_{0}$, and $\rho$ behave essentially as constants. Moreover, there is a constant $C_{4}$ such that for $\psi \in I$,

$$
\left|w_{0}(\psi)\right| \leq C_{4}|I|^{2}
$$

Thus we have

$$
\begin{gathered}
w(I) \sim|I|, \\
\rho(I)^{2} \sim|I|^{2}, \\
\left\|\lambda_{n}\left(w w_{0}\right)^{1 / 2}\right\|_{2}^{2} \lesssim|I|^{2}, \\
\left\|\lambda_{n}\left(w_{0}^{1 / 2} \rho_{0}\right)\right\|_{2}^{2} \lesssim|I|, \\
\left\|\lambda_{n}\left(w_{0}^{1 / 2} \rho\right)\right\|_{2}^{2} \lesssim|I|^{2}, \\
\left\|\lambda_{n}\left(\rho \rho_{0}\right)\right\|_{2}^{2} \lesssim|I|
\end{gathered}
$$

so that, combining (6.72) and (6.85)-(6.91) we have

$$
\int_{I}\left|\left\{\left[M_{\beta}, \tilde{S}_{n}(0)\right] f_{\theta}\right\}(\theta)\right|^{2} d \theta \leq K_{3} C^{2} w(I) \rho(I)^{2}
$$

where $K_{3}$ is independent of $\beta, I, n$. Combining (6.56) and (6.92), we have

$$
w(I)^{-1} \int_{I}\left|\beta(\theta)-c_{I}(\beta)\right|^{2} w(\theta) d \theta \leq 2 \pi^{2} K_{3} C^{2} .
$$

Case (iv): $\pi / 200>2|I| \geq \pi-\psi_{0}$. This case is in certain respects analogous to Case (i). Using the fact that $\sin x=\sin (\pi-x)$, we write, as in (6.57), (6.94)

$$
\begin{aligned}
\sin \left(\frac{\theta+\psi}{2}\right) \sin \left(\frac{\theta-\psi}{2}\right) & =\sin \left[\frac{(\pi-\theta)+(\pi-\psi)}{2}\right] \sin \left[\frac{(\pi-\theta)-(\pi-\psi)}{2}\right] \\
& =w_{\pi}(\theta) \rho_{\pi}^{2}(\psi)-w_{\pi}(\psi) \rho_{\pi}^{2}(\theta)
\end{aligned}
$$


where $w_{\pi}(\psi)=w(\pi-\psi), \rho_{\pi}(\psi)=\rho(\pi-\psi)$. Thus we obtain (6.60) with $w_{\pi}$ in place of $w$ and $\rho_{\pi}$ in place of $\rho$. The estimate involving $w_{\pi}$ and $\rho_{\pi}$ are essentially the same as those involving $w$ and $\rho$ in Case (i). Moreover, it is easy to see that $w(I) \sim|I|$ while $\rho(I)^{2} \gtrsim|I|^{4}$, so that $w(I) \rho(I)^{2} \gtrsim|I|^{5}$. Thus, by essentially the same argument as in Case (i), we have

$$
w(I)^{-1} \int_{I}\left|\beta(\theta)-c_{I}(\beta)\right|^{2} w(\theta) d \theta \leq 2 \pi^{2} K_{4} C^{2}
$$

where $K_{4}$ is independent of $\beta, I, n$.

Case (v): $\pi / 200>\pi-\psi_{0}>2|I|$. This case is analogous to Case (ii). We write

$$
\sin \left(\frac{\theta-\psi}{2}\right)=w_{0}^{1 / 2}(\theta) \rho_{0}(\psi)-w_{0}^{1 / 2}(\psi) \rho_{0}(\theta)
$$

$$
\sin \left(\frac{\theta+\psi}{2}\right)=\sin \left[\frac{(\pi-\theta)}{2}+\frac{(\pi-\psi)}{2}\right]=w_{\pi}^{1 / 2}(\theta) \rho_{\pi}(\psi)+\rho_{\pi}(\theta) w_{\pi}^{1 / 2}(\psi)
$$

and, as in Case (ii), we obtain (6.72) with $w_{\pi}$ in place of $w$ and $\rho_{\pi}$ in place of $\rho$.

Geometrically, $I$ is a "mirror image" across $\psi=\pi / 2$ of an interval of the type considered in Case (ii). Consequently, we obtain estimates of the form

$$
\begin{gathered}
\rho(I) \gtrsim\left(\pi-\psi_{0}\right)|I|, \quad w(I) \sim|I|, \\
\left|\left(w_{\pi} w_{0}\right)^{1 / 2}(\psi)\right| \lesssim|I| \quad \text { for } \psi \in I, \\
\left|\left(w_{\pi}^{1 / 2} \rho_{0}\right)(\psi)\right| \leq 1 \quad \text { for } \psi \in I, \\
\left|\left(w_{0}^{1 / 2} \rho_{\pi}\right)(\psi)\right| \lesssim\left(\pi-\psi_{0}\right)|I| \text { for } \psi \in I, \\
\left|\left(\rho_{\pi} \rho_{0}\right)(\psi)\right| \lesssim\left(\pi-\psi_{0}\right) \quad \text { for } \psi \in I
\end{gathered}
$$

so that

$$
\begin{gathered}
\left\|\lambda_{n}\left(w_{\pi} w_{0}\right)^{1 / 2}\right\|_{2}^{2} \lesssim|I|^{3}, \\
\left\|\lambda_{n}\left(w_{\pi}^{1 / 2} \rho_{0}\right)\right\|_{2}^{2} \leq|I|, \\
\left\|\lambda_{n}\left(w_{0}^{1 / 2} \rho_{\pi}\right)\right\|_{2}^{2} \lesssim\left(\pi-\psi_{0}\right)^{2}|I|^{3} \\
\left\|\lambda_{n}\left(\rho_{\pi} \rho_{0}\right)\right\|_{2}^{2} \lesssim\left(\pi-\psi_{0}\right)^{2}|I| .
\end{gathered}
$$

Thus we have

$$
\int_{I}\left|\left\{\left[M_{\beta}, \tilde{S}_{n}(0)\right] f_{\theta}\right\}(\theta)\right|^{2} d \theta \leq K^{\prime} C^{2}|I|^{3}\left(\pi-\psi_{0}\right)^{2} \leq K_{5} C^{2} w(I) \rho(I)^{2}
$$

where $K^{\prime}, K_{5}$ are independent of $\beta, I, n$. Combining (6.56) and (6.107), we obtain

$$
w(I)^{-1} \int_{I}\left|\beta(\theta)-c_{I}(\beta)\right|^{2} w(\theta) d \theta \leq 2 \pi^{2} K_{5} C^{2} .
$$


We have now considered all possible cases. If we take $\delta=2 \pi^{2} \max _{1 \leq j \leq 5} K_{j}$, we obtain (6.48), and the proof is complete.

Corollary 6.5.1. $\mathrm{BMO}_{e}$ is the space of uniform holomorphy at 0 for $\left\langle\widetilde{P}_{n}\right\rangle$ and $\left\langle\widetilde{Q}_{n}\right\rangle$.

We also obtain the following result for partial sum operators on $[-1,1]$ :

Corollary 6.5.2. In the notation of $\S 4$, BMO is the space of uniform holomorphy at 0 for $\left\langle P_{n}^{-1 / 2,-1 / 2}\right\rangle$ and $\left\langle Q_{n}^{-1 / 2,-1 / 2}\right\rangle$.

Proof. By Corollary 4.2.1, it suffices to show that

$$
C=\sup _{n} \|\left[M_{\beta}, S_{n}^{-1 / 2,-1 / 2}(0) \|_{\mathscr{L}_{-1 / 2,-1 / 2}(0)}<\infty,\right.
$$

for $\beta \in L^{1}\left([-1,1],\left(1-x^{2}\right)^{-1 / 2} d x\right)$, only if $\beta \in$ BMO.

For $f \in L_{-1 / 2,-1 / 2}(0)$ and $\theta \in[0, \pi]$, define $U f(\theta)=f \circ \cos (\theta)$. It is easy to see that $U$ is an isometry from $L_{-1 / 2,-1 / 2}(0)$ to $\tilde{L}(0)$. By virtue of this isometry, we see that the family $\left\langle t_{n}: n \in \mathbf{N}\right\rangle$ of Chebyshev polynomials, defined by

$$
t_{n}(x)=\cos n \theta, \quad x=\cos \theta
$$

is an orthogonal polynomial system on $[-1,1]$ relative to $\left(1-x^{2}\right)^{-1 / 2}$ (cf. [10], $\S \S 1.12$ and 2.4). In particular, we see that

$$
S_{n}^{-1 / 2,-1 / 2}(0)=U^{-1} \widetilde{S}_{n}(0) U
$$

so that

$$
\left[M_{\beta}, S_{n}^{-1 / 2,-1 / 2}(0)\right]=U^{-1}\left[M_{\beta \circ \cos }, \widetilde{S}_{n}(0)\right] U .
$$

Since $U, U^{-1}$ are isometries, (6.109) and (6.112) imply that $\beta \circ \cos \in \mathbf{B M O}_{e}$.

Now suppose $I=[a, b]$ is any subinterval of $[-1,1]$, and let $\omega(x)=$ $\left(1-x^{2}\right)^{-1 / 2}$; clearly $\omega$ is an $A_{\infty}$ weight on $[-1,1]$. Let $J=[\arccos b, \arccos a]$; then we have

$$
\begin{aligned}
\omega(I)^{-1} \int_{I} \mid \beta(x) & -m_{J}(\beta \circ \cos ) \mid \omega(x) d x \\
= & |J|^{-1} \int_{J}\left|\beta \circ \cos (\theta)-m_{J}(\beta \circ \cos )\right| d \theta \leq\|\beta \circ \cos \|_{*} .
\end{aligned}
$$

Thus $\beta \in$ BMO by the $[-1,1]$-version of Lemma 6.3 .

\section{REFERENCES}

1. M. S. Berger, Nonlinearity and functional analysis: lectures on nonlinear problems in mathematical analysis, Academic Press, New York, 1977.

2. R. R. Coifman and R. Rochberg, Projections in weighted spaces, skew projections and inversion of Toeplitz operators, Integral Equations Operator Theory 5 (1982), 142-159.

3. R. R. Coifman, R. Rochberg and G. Weiss, Factorization theorems for Hardy spaces in several variables, Ann. of Math. 103 (1976), 611-635. 
4. P. Deift, L. C. Li and C. Tomei, Toda flows with infinitely many variables, J. Funct. Anal. 64 no. 3 (1985), 358-402.

5. J. Garcia-Cuerva and J. L. Rubio de Francia, Weighted norm inequalities and related topics, North-Holland Math. Studies \#116/Notas de Matemáticas 104, North-Holland, Amsterdam, 1985.

6. J. B. Garnett, Bounded analytic functions, Academic Press, New York, 1981.

7. R. Hunt, B. Muckenhoupt and R. Wheeden, Weighted norm inequalities for the conjugate function and Hilbert transform, Trans. Amer. Math. Soc. 176 (1973), 227-251.

8. N. Kerzman and E. M. Stein, The Szegö kernel in terms of Cauchy-Fantappie kernels, Duke Math. J. 45 (1978), 197-224.

9. B. Muckenhoupt, Mean convergence of Jacobi series, Proc. Amer. Math. Soc. 23 (1969), 306310.

10. G. Szegö, Orthogonal polynomials, 4th ed., Amer. Math. Soc., Providence, R.I., 1975.

Department of Mathematics, Yale University, New Haven, Connecticut 06520

Department of Mathematics, Virginia Polytechnic Institute and State University, BLACKSBURG, Virginia 24061-0123 